\title{
QUANTITATIVE ESTIMATES ON THE SINGULAR SETS OF ALEXANDROV SPACES
}

\author{
NAN LI AND AARON NABER
}

\begin{abstract}
Let $X \in \operatorname{Alex}^{n}(-1)$ be an $n$-dimensional Alexandrov space with curvature $\geq-1$. Let the $r$-scale $(k, \epsilon)$-singular set $\mathcal{S}_{\epsilon, r}^{k}(X)$ be the collection of $x \in X$ so that $B_{r}(x)$ is not $\epsilon r$-close to a ball in any splitting space $\mathbb{R}^{k+1} \times Z$. We show that there exists $C(n, \epsilon)>0$ and $\beta(n, \epsilon)>0$, independent of the volume, so that for any disjoint collection $\left\{B_{r_{i}}\left(x_{i}\right): x_{i} \in \mathcal{S}_{\epsilon, \beta r_{i}}^{k}(X) \cap B_{1}, r_{i} \leq 1\right\}$, the packing estimate $\sum r_{i}^{k} \leq C$ holds. Consequently, we obtain the Hausdorff measure estimates $\mathcal{H}^{k}\left(\mathcal{S}_{\epsilon}^{k}(X) \cap B_{1}\right) \leq C$ and $\mathcal{H}^{n}\left(B_{r}\left(\mathcal{S}_{\epsilon, r}^{k}(X)\right) \cap B_{1}(p)\right) \leq C r^{n-k}$. This answers an open question in [8]. We also show that the $k$-singular set $\mathcal{S}^{k}(X)=\underset{\epsilon>0}{\cup}\left(\underset{r>0}{\cap} \mathcal{S}_{\epsilon, r}^{k}\right)$ is $k$-rectifiable and construct examples to show that such a structure is sharp. For instance, in the $k=1$ case we can build for any closed set $T \subseteq \mathbb{S}^{1}$ and $\epsilon>0$ a space $Y \in \operatorname{Alex}^{3}(0)$ with $\mathcal{S}_{\epsilon}^{1}(Y)=\phi(T)$, where $\phi: \mathbb{S}^{1} \rightarrow Y$ is a bi-Lipschitz embedding. Taking $T$ to be a Cantor set it gives rise to an example where the singular set is a 1-rectifiable, 1-Cantor set with positive 1-Hausdorff measure.
\end{abstract}

\section{CONTENTS}

1. Introduction 2

2. Outline of the Proof $\quad 5$

3. Monotonicity and Packing Numbers 6

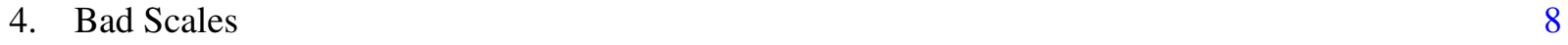

5. Splitting Theory and Dimension Reduction 13

5.1. Splitting theory 13

5.2. Strong and weak singularity 15

5.3. Dimension reduction 17

6. Packing Estimates 19

7. Sharpness of the Rectifiability 24

$\begin{array}{ll}\text { References } & 27\end{array}$

The first author was supported by PSC-CUNY Grant 61533-0049. The second author was supported by NSF grant DMS1809011. 


\section{INTRODUCTION}

Let $\operatorname{Alex}^{n}(\kappa)$ be the collection of $n$-dimensional Alexandrov spaces with (sectional) curvature $\geq \kappa$. The aim of this paper is to study the quantitative stratifications of $X \in \operatorname{Alex}^{n}(\kappa)$. Given $X \in \operatorname{Alex}^{n}(\kappa)$, it is known that the tangent cone $T_{p}(X)$ at every point $p \in X$ is a metric cone $C(\Sigma)$, where $\Sigma \in \operatorname{Alex}^{n-1}(1)$ and it is unique. The singular set $\mathcal{S}(X)$ is the collection of points whose tangent cones are not isometric to $\mathbb{R}^{n}$. It has a natural stratification

$$
\mathcal{S}(X)=\mathcal{S}^{n-1}(X) \supseteq \mathcal{S}^{n-2}(X) \supseteq \cdots \supseteq \mathcal{S}^{1}(X) \supseteq \mathcal{S}^{0}(X),
$$

where

$$
\mathcal{S}^{k}(X) \equiv\left\{p \in X: T_{p}(X) \text { is not isometric to } \mathbb{R}^{k+1} \times C(\Sigma) \text { for any metric space } \Sigma\right\}
$$

We may omit the $X$ and write for example $\mathcal{S}^{k}=\mathcal{S}^{k}(X)$ if it doesn't cause any ambiguity. Let us first state a notion of strong quantitative singular sets. We will then compare it with those used for the Ricci cases.

Definition 1.1 (Quantitative splitting).

(1) Given a metric space $Y$ and $k \in \mathbb{N}$, we say that $Y$ is $k$-splitting if $Y$ is isometric to $\mathbb{R}^{k} \times Z$ for some metric space $Z$.

(2) Given a metric space $X$ we say that a metric ball $B_{r}(x) \subseteq X$ is $(k, \epsilon)$-splitting if there exists a $k$ splitting space $Y$ and $y \in Y$ such that $d_{G H}\left(B_{r}(x), B_{r}(y)\right) \leq \epsilon r$.

Definition 1.2 (Strong quantitative singular sets). Given $k, \epsilon, r>0$ and metric space $X$.

(1) The $r$-scale $(k, \epsilon)$-singular set

$$
\mathcal{S}_{\epsilon, r}^{k}(X) \equiv\left\{x \in X: B_{r}(x) \text { is not }(k+1, \epsilon) \text {-splitting }\right\} .
$$

(2) The $(k, \epsilon)$-singular set

$$
\mathcal{S}_{\epsilon}^{k} \equiv \underset{r>0}{\cap} \mathcal{S}_{\epsilon, r}^{k}=\left\{x \in X: B_{r}(x) \text { is not }(k+1, \epsilon) \text {-splitting for every } 0<r \leq 1\right\} .
$$

It's easy to see that $\mathcal{S}^{k}=\underset{\epsilon>0}{\cup} \mathcal{S}_{\epsilon}^{k}=\underset{\epsilon>0}{\cup}\left(\underset{r>0}{\cap} \mathcal{S}_{\epsilon, r}^{k}\right)$. A weaker notion of quantitative singular sets, which we will denote by $\mathcal{W} \mathcal{S}_{\epsilon, r}^{k}$, was introduced in [5] for manifolds with lower Ricci curvature bounds, see (5.10) for a definition. A significance for (1.2) is that it requires $B_{s}(x)$ to be $(k, \epsilon)$-non-splitting only at the scale $s=r$, but not for all $r \leq s \leq 1$ as required in (5.10). It is worth pointing out that notion (1.2) is strictly stronger than (5.10) on manifolds with Ricci curvature bounds, while they are equivalent in some sense on Alexandrov spaces (see Section 5.2). The singular sets defined as in (1.2) are not known to satisfy the estimates established in [4], [5] or [6] for the Ricci cases.

It was proved in [3] that if $X$ is a Gromov-Hausdorff limit of $n$-dimensional, $v$-noncollapsed Riemannian

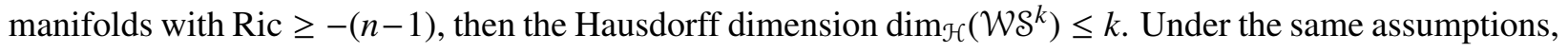
it was proved in [4] that for any $0<r, \epsilon \leq 1$, there exists a constant $C(n, v, \epsilon)>0$ such that for any $p \in X$, it holds that

$$
\operatorname{vol}\left(B_{r}\left(\mathcal{W S}_{\epsilon, r}^{k}(X)\right) \cap B_{1 / 2}(p)\right) \leq C(n, v, \epsilon) r^{n-k}
$$


It was also proved in [4] that $\mathcal{W} \mathcal{S}_{\epsilon}^{k}(X)$ is $k$-rectifiable. For $X \in \operatorname{Alex}^{n}(\kappa)$, it is proved in [2] that the Hausdorff dimension $\operatorname{dim}_{\mathcal{H}}\left(\mathcal{S}^{k}(X)\right) \leq k$, and it was asked in [8] wether the $(n-2)$-dimensional packing estimate holds for $\mathcal{S}_{\epsilon}^{n-2}(X)$. In this paper, we prove the $k$-packing estimates and the $k$-rectifiability of $\mathcal{S}_{\epsilon}^{k}(X)$ for every $0 \leq k \leq n$. Moreover, all of our estimate are independent on the volume of unit balls in $X$. Note that it is crucial to have a positive lower volume bound in [4], [5] and [6], to obtain estimates such as (1.4) for manifolds with lower Ricci curvature bounds. It is not known whether the volume dependence can be removed for the Ricci cases.

Theorem 1.3 (Packing estimate). For any $n \in \mathbb{N}$ and $\epsilon>0$ there exists $C=C(n, \epsilon)>0$ and $\beta=\beta(n, \epsilon)>0$ such that the following hold for any $(X, p) \in$ Alex $^{n}(-1)$. If $x_{i} \in \mathcal{S}_{\epsilon, \beta r_{i}}^{k}(X) \cap B_{1}(p)$ and $\left\{B_{r_{i}}\left(x_{i}\right)\right\}$ are disjoint with $r_{i} \leq 1$ for all $i \in \mathbb{I}$, then

$$
\sum_{i \in \mathbb{I}} r_{i}^{k}<C
$$

In particular, if $x_{i} \in \mathcal{S}_{\epsilon, r}^{k}(X) \cap B_{1}(p)$ and $\left\{B_{r}\left(x_{i}\right)\right\}$ are disjoint with $r \leq 1$, then $|\mathbb{I}|<C r^{-k}$.

Example 1.1. There exists Alexandrov spaces (in fact non-collapsed Gromov-Hausdorff limits of manifolds with sec $\geq 0$ ) whose singular set is dense. Such a space was constructed in [9]. Begin with a regular tetrahedron $X_{1}$ in $\mathbb{R}^{3}$. Suppose convex polyhedra $X_{k}$ with triangular faces $\Delta_{i}, i=1,2 \ldots, 4 \cdot 3^{k-1}$ has been constructed. Let $x_{i}$ be the centroid of face $\Delta_{i}$. Let $y_{i} \in \mathbb{R}^{3}$ so that $d\left(y_{i}, X_{k}\right)=d\left(y_{i}, x_{i}\right)=d_{k}^{i}>0$. Let $Y_{i}$ be the tetrahedron formed by $y^{i}$ and $\Delta_{i}$. Define $X_{k+1}=X_{k} \cup\left(\cup_{i} Y_{i}\right)$. The constants $d_{k}^{i}=d_{k}^{i}\left(X_{k}\right)$ can be chosen small enough so that $X_{k+1}$ is convex. We have that $\partial X_{k} \in \operatorname{Alex}^{2}(0)$ for all $k$. Thus $Y=\lim _{i \rightarrow \infty} \partial X_{k} \in \operatorname{Alex}^{2}(0)$. It's easy to see that if all $X_{k}$ are convex, then $\max _{i}\left\{d_{k}^{i}\right\} \rightarrow 0$ as $k \rightarrow \infty$.

The set of singular points $\mathcal{S}^{0}(Y) \supseteq \bigcup_{i, k}\left\{x_{k}^{i}\right\}$ is dense in $Y$. However, $\left|\mathcal{S}_{\epsilon}^{0}\right|<N(\epsilon)$, asserted by Theorem 1.3. For this example, we can get an explicit estimate using Gauss-Bonnet formula. For each $p \in Y$, we have that the tangent cone $T_{p}(Y)=C\left(\mathbb{S}_{\beta}^{1}\right)$ with $0<\beta \leq 1$. Let $\theta_{p}=2 \pi \beta$ be the cone angle. Then we have $\mathcal{S}_{\epsilon}^{0}=\left\{p \in Y: \theta_{p} \leq 2 \pi-\epsilon\right\}$. Note that for any $p \in Y$ the Gaussian curvature $K_{p} \geq 0$ and $K_{p}=\left(2 \pi-\theta_{p}\right) \delta_{p}$ if $p \in \mathcal{S}_{\epsilon}^{0}$, where $\delta_{p}$ is the Dirac delta function at $p$. By Gauss-Bonnet formula, we have for $\epsilon_{i}=2^{-i}$

$$
4 \pi=\int_{Y} K \geq \sum_{i=0}^{\infty} \sum_{p \in \mathcal{S}_{\epsilon_{i+1}}^{0} \backslash \mathcal{S}_{\epsilon_{i}}^{0}}\left(2 \pi-\theta_{p}\right) \geq \sum_{i=0}^{\infty} \epsilon_{i+1}\left|\mathcal{S}_{\epsilon_{i+1}}^{0} \backslash \mathcal{S}_{\epsilon_{i}}^{0}\right| .
$$

In particular, we have the estimate $\left|\mathcal{S}_{\epsilon}^{0}\right| \leq \frac{4 \pi}{\epsilon}$.

The statement of Theorem 1.3 (ii) is not true without a quantitative control of $\beta=\beta(n, \epsilon)$, if inf $\left\{r_{i}\right\}=0$. See the following example.

Example 1.2. Let $X=C\left(S_{\rho}^{1}\right)$ be a metric cone over a circle with radius $\rho=\frac{1}{20}$. Let $p$ be the cone point and choose points $x_{i} \in X$, so that $d\left(p, x_{i}\right)=3^{-i}, i=0,1,2, \ldots$ Consider disjoint collection $\mathcal{C}=\left\{B_{r_{i}}\left(x_{i}\right): r_{i}=\right.$ $\left.\frac{1}{2} \cdot 3^{-i}\right\}$. By the cone structure, we have $d_{G H}\left(B_{r_{i}}\left(x_{i}\right), Z \times\left[-r_{i}, r_{i}\right]\right) \geq \frac{1}{10} r_{i} \sin (\pi \rho)>\frac{1}{100} r_{i}$ for any metric space $Z$. Thus $x_{i} \in \mathcal{S}_{\epsilon, r_{i}}^{0}(X)$ for any $0<\epsilon<\frac{1}{200}$. However, $|\mathcal{C}|=\infty$.

By a standard covering technique, Theorem 1.3 implies the following Hausdorff measure estimate. 
Corollary 1.4 (Hausdorff measure estimate). For any $n \in \mathbb{N}$ and $\epsilon>0$ there exists $C=C(n, \epsilon)>0$ such that for any $X \in$ Alex $^{n}(-1)$ and $p \in X$, we have the Hausdorff measure estimate

$$
\mathcal{H}^{k}\left(\mathcal{S}_{\epsilon}^{k}(X) \cap B_{1}(p)\right)<C(n, \epsilon) .
$$

We also have the following conjectural form of the constant in the above theorem:

Conjecture 1.1. For any $(X, p) \in \operatorname{Alex}^{n}(-1)$, we have

$$
\mathcal{H}^{k}\left(\mathcal{S}_{\epsilon}^{k} \cap B_{1}(p)\right)<C(n) \epsilon^{1-(n-k)} .
$$

Indeed, we may even have the following stronger summable form, see Example 1.1:

Conjecture 1.2. For any $(X, p) \in \operatorname{Alex}^{n}(-1)$ and let $\epsilon_{i}=2^{-i}$, then we have

$$
\sum_{i=0}^{\infty} \epsilon_{i+1}^{(n-k)-1} \mathcal{H}^{k}\left(\left(\mathcal{S}_{\epsilon_{i+1}}^{k} \backslash \mathcal{S}_{\epsilon_{i}}^{k}\right) \cap B_{1}(p)\right)<C(n)
$$

Now let $\left\{B_{r}\left(x_{i}\right)\right\}_{i=1}^{N}$ be a Vitali covering of $B_{r}\left(\mathcal{S}_{\epsilon, r}^{k}(X) \cap B_{1}(p)\right)$ with $x_{i} \in \mathcal{S}_{\epsilon, r}^{k}(X)$. By Theorem 1.3, we have that $N \leq C(n, \epsilon) r^{-k}$. Combining it with $\mathcal{H}^{n}\left(B_{r}(x)\right) \leq C(n) r^{n}$ for every $x \in X$ and $r \leq 1$, we have the following estimate, which only matters in the noncollapsing setting:

Corollary 1.5 (Volume estimate). For any $n \in \mathbb{N}$ and $\epsilon>0$ there exists $C=C(n, \epsilon)>0$ such that the following estimate holds for any $X \in \operatorname{Alex}^{n}(-1)$ and $p \in X$.

$$
\mathcal{H}^{n}\left(B_{r}\left(\mathcal{S}_{\epsilon, r}^{k}(X)\right) \cap B_{1}(p)\right) \leq C r^{n-k} .
$$

We also show that $\delta_{\epsilon}^{k}$ is $k$-rectifiable.

Theorem 1.6 ( $k$-rectifiability). For any $X \in$ Alex $^{n}(-1)$ and $0 \leq k \leq n$ we have that $\mathcal{S}^{k}(X)$ is $k$-rectifiable.

It was asked for both Ricci and Alexandrov cases wether $\mathcal{S}_{\epsilon}^{k}$ carries with a $k$-manifold structure, away from a zero $\mathcal{H}^{k}$-measure subset. It was proved in [2] that for any $X \in \operatorname{Alex}^{n}(\kappa)$, if $p \in X \backslash \mathcal{S}_{\epsilon}^{n-1}$, then there exists $r>0$ so that $B_{r}(p)$ is bi-Lipschitz to $B_{r}(0) \subset \mathbb{R}^{n}$. If $p \in \mathcal{S}_{\epsilon}^{n-1} \backslash \mathcal{S}_{\epsilon}^{n-2}$, then there exists $r>0$ so that $B_{r}(p)$ is bi-Lipschitz to a ball centered at the origin in the half space $\mathbb{R}^{n-1} \times \mathbb{R}_{\geq 0}$. For $\mathcal{S}_{\epsilon}^{n-2}$, we construct examples $X \in \operatorname{Alex}^{n}(\kappa)$ to show that it may contain no manifold point.

Theorem 1.7. For any closed subset $T \subseteq \mathbb{S}^{1}$ and $\epsilon>0$, there exists a sequence of 3-dimensional manifolds $M_{i}$ with $\sec _{M_{i}} \geq 0$ and $M_{i} \rightarrow Y \in$ Alex $^{3}(0)$, for which $\mathcal{S}_{\epsilon}^{1}(Y)=\phi(T)$, where $\phi: \mathbb{S}^{1} \rightarrow Y$ is a bi-Lipschitz embedding.

In particular, $\mathcal{S}_{\epsilon}^{1}(Y)$ can be a Cantor set with $\mathcal{H}^{1}\left(\mathcal{S}_{\epsilon}^{1}(Y)\right)>0$, which contains no manifold points. Let $Y_{n}=Y \times \mathbb{R}^{n-3} \in \operatorname{Alex}^{n}(0)$. We have that $\mathcal{S}_{\epsilon}^{n-2}\left(Y_{n}\right)$ contains no $(n-2)$-dimensional manifold point. This shows that the rectifiable structure in Theorem 1.6 is sharp. Examples for which $\mathcal{S}_{\epsilon}^{k}$ contains no $k$-manifold point, where $n \geq 4$ and $1 \leq k \leq n-3$, can be similarly constructed, with a good amount of extra technical work. 


\section{Outline of The Proof}

We begin with the notion of bad scales $\operatorname{Bad}^{\epsilon}(p)$. Fix a point $p \in X$ and $\epsilon>0$, then we define a $\mathbb{Z}_{2}$ valued function $T_{p}^{\epsilon}(r, R)$ to describe the symmetry of metric balls $B_{s}(p)$ over scales $0 \leq r \leq s \leq R$. Define $T_{p}^{\epsilon}(r, R)=0$ if there exists a cone space $C(\Sigma)$, depending on $p, r, R, \epsilon$ but not on $s \in[r, R]$, so that

$$
d_{G H}\left(B_{s}(p), B_{s}\left(p^{*}\right)\right) \leq \epsilon s,
$$

for every $s \in[r, R]$, where $p^{*} \in C(\Sigma)$ is the cone point. Otherwise we define $T_{p}^{\epsilon}(r, R)=1$. In the case that $T_{p}^{\epsilon}(r, R)=0$, we say that the metric ball $B_{s}(p)$ is uniformly $(0, \epsilon)$-symmetric for $r \leq s \leq R$. It is clear that if $\left[a_{1}, a_{2}\right] \subseteq[r, R]$ and $T_{p}^{\epsilon}(r, R)=0$, then $T_{p}^{\epsilon}\left(a_{1}, a_{2}\right)=0$. Contrapositively, if we have $[r, R] \subseteq\left[b_{1}, b_{2}\right]$ with $T_{p}^{\epsilon}(r, R)=1$, then we also have $T_{p}^{\epsilon}\left(b_{1}, b_{2}\right)=1$.

Definition 2.1 (Bad scales). Let $r_{\alpha}=2^{-\alpha}$, where $\alpha \in \mathbb{N}$. The $\epsilon$-bad scales $\left\{r_{\beta_{(j)}}\right\} \subseteq\left\{r_{\alpha}, \alpha \in \mathbb{N}\right\}$ of $p$, denoted by $\operatorname{Bad}^{\epsilon}(p)$, are defined recursively as follows. Let $r_{\beta_{(0)}}=r_{0}=1$ and

$$
r_{\beta_{(k+1)}}= \begin{cases}r_{\beta_{(k)}+1}, & \text { if } \quad T_{p}^{\epsilon}\left(r_{\beta_{(k)}+1}, r_{\beta_{(k)}}\right)=1 ; \\ r_{\alpha}, & \text { if there exists } \alpha \geq \beta_{(k)}+1 \text { such that } T_{p}^{\epsilon}\left(r_{\alpha}, r_{\beta_{(k)}}\right)=0 \text { but } T_{p}^{\epsilon}\left(r_{\alpha+1}, r_{\beta_{(k)}}\right)=1 .\end{cases}
$$

Note that if $[r, R]$ contains no $\epsilon$-bad scale of $p$, then $B_{s}(p)$ is uniformly $(0, \epsilon)$-symmetric for $r \leq s \leq R$. This definition is strictly stronger than the corresponding definitions in the Ricci curvature context.

The following is a key lemma to build up our covering techniques.

Lemma 2.2 (Finiteness of the number of bad scales). For any $n \in \mathbb{N}$ and $\epsilon>0$, there exists $N(n, \epsilon)>0$ such that for any $(X, p) \in$ Alex $^{n}(-1)$, the number of $\epsilon$-bad scales satisfies $\left|\operatorname{Bad}^{\epsilon}(p)\right|<N(n, \epsilon)$.

The proof of this lemma is based on various point-wise monotonic properties of Alexandrov spaces. In particular, we prove Lemma 4.3, which we call "almost packing cone implies almost metric cone". It is an analogy of "almost volume cone implies almost metric cone", which is the monotonic formula used for manifolds with lower Ricci curvature bound. Note that both our monotonicity and the corresponding rigidity are strictly stronger than in the Ricci curvature context.

In order to state and prove our rigidity results we will need a splitting theory for Alexandrov spaces.

Definition 2.3 (Strong splitting maps). Let $u_{1}, u_{2}, \ldots, u_{k}: B_{R}(p) \rightarrow \mathbb{R}$ be $\epsilon$-concave functions. The map $u=\left(u_{1}, \ldots, u_{k}\right): B_{R}(p) \rightarrow \mathbb{R}^{k}$ is called a $(k, \epsilon)$-splitting map if the following are satisfied.

(i) $\left|\left\langle\nabla u_{i}, \nabla u_{j}\right\rangle-\delta_{i j}\right| \leq \epsilon$.

(ii) For any $x, y \in B_{R}(p)$ and any minimizing geodesic $\gamma$ connecting $x$ and $y$, it holds that

$$
\left\langle\uparrow_{x}^{y}, \nabla_{x} u_{i}\right\rangle+\left\langle\uparrow_{y}^{x}, \nabla_{y} u_{i}\right\rangle \leq \epsilon
$$

Here $\uparrow_{x}^{y}$ and $\uparrow_{y}^{x}$ denote the unit tangent directions of $\gamma$ at $x$ and $y$ respectively.

Remark 2.1. If $X$ is a smooth Riemannian manifold, the condition (ii) in the above definition says that on each geodesic, $u$ has a lower integral hessian bound. 
By the definition, we have that if $u: B_{R}(p) \rightarrow \mathbb{R}^{k}$ is a $(k, \epsilon)$-splitting map, then $\left.u\right|_{B_{r}}$ is also a $(k, \epsilon)$-splitting map for any $B_{r} \subset B_{R}(p)$. This restriction property of splitting maps is false in the context of manifolds with lower Ricci curvature bounds. The existence and the properties of the strong splitting maps are discussed in Section 5.1.

As in the standard dimension reduction, let us observe that for a metric cone $C(Z)$, the tangent cone of any point away from the cone tip splits off an extra $\mathbb{R}$-factor comparing to $C(Z)$. We prove an effective version of this property in Lemma 5.7.

The monotonic property and the splitting theory lead to Theorem 6.2. It says that there exist $\delta(n, \epsilon)$ and $\beta(n, \epsilon)>0$ so that if $u: B_{50}(p) \rightarrow \mathbb{R}^{k}$ is a $(k, \delta)$-splitting function, and $\left\{B_{r_{i}}\left(x_{i}\right)\right\}$ is a disjoint collection with $x_{i} \in \mathcal{S}_{\epsilon, \beta r_{i}}^{k}$, then for any $z \in \mathbb{R}^{k}$, we have

$$
\left|\left\{i \in \mathbb{I}: B_{\beta r_{i}}\left(x_{i}\right) \cap u^{-1}(z) \neq \varnothing\right\}\right|<N(n, \epsilon) .
$$

In particular, this Theorem implies that if we look at the associated collection of balls $\left\{B_{\beta r_{i} / 4}\left(u\left(x_{i}\right)\right)\right\} \subseteq \mathbb{R}^{k}$ then its intersection number is at most $N(n, \epsilon)$. That is, given any ball $B_{\beta r_{j} / 4}\left(u\left(x_{j}\right)\right) \in\left\{B_{\beta r_{i} / 4}\left(u\left(x_{i}\right)\right)\right\}$ it intersects at most $N-1$ other balls from the collection. This shows that Theorem 1.3 holds if $B_{1}(p)$ is $(k, \epsilon)$-splitting. We will then complete the proof by an induction on $k$.

In Section 7 we construct examples to prove Theorem 1.7. Let us explain the moral of the construction below. The technical details will be added to make it rigorous in Section 7.

Let $Z=\bar{B}_{1} \subset \mathbb{R}^{2}$ be a closed unit disk and $X_{0}=Z \times[0,1] \in$ Alex $^{3}(0)$ be a solid cylinder. For $\epsilon>0$ small, we have $\mathcal{S}^{0}\left(X_{0}\right)=\varnothing$ and $\mathcal{S}_{\epsilon}^{1}\left(X_{0}\right)=\partial Z \times\{0,1\}$ is a union of two unit circles.

Now let $T \subseteq \partial Z$ be a closed subset, and thus $\partial Z \backslash T=\cup_{\ell} U_{\ell}$ is a collection of disjoint open intervals. Let $p$ be the center of $Z$ and define $C_{\ell}=\cup_{x \in U_{\ell}} \gamma_{p x}$, where $\gamma_{x, y}$ denotes a line connecting $x$ and $y$, be the collection of sectors associated to the open sets $U_{\ell}$. Let us observe for any $x \in \partial Z$ that the curvature at $(x, 1) \in X_{0}$ is $+\infty$ along the normal direction of $\partial Z \times\{1\}$ and strictly positive along its tangential direction. This will allow us to smoothly "sand off" each of $U_{\ell} \times\{1\}$ inside its convex hull $C_{\ell} \times[0,1]$, so that both the convexity of $X_{0}$ and the tangent cones at points in $X_{0} \backslash\left(\cup C_{\ell} \times[0,1]\right)$ are preserved. Let $X_{1} \in$ Alex $^{3}(0)$ be the resulted space. In particular, the tangent cones at the points of $T \times\{1\}$ are preserved, and thus we have that $\mathcal{S}_{\epsilon}^{1}\left(X_{1}\right)=(T \times\{1\}) \cup(\partial Z \times\{0\})$. Similarly, we can smooth near $\partial Z \times\{0\}$ in order to construct $X_{2}$ with $S_{\epsilon}^{1}\left(X_{2}\right)=T \times\{1\}$. Now let $Y_{2}$ be the doubling of $X_{2}$, which is now a boundary free Alexandrov space $Y_{2} \in \operatorname{Alex}^{3}(0)$ for which $\mathcal{S}\left(Y_{2}\right)=\mathcal{S}_{\epsilon}^{1}\left(Y_{2}\right)=T$ and $\mathcal{S}_{\epsilon}^{0}\left(Y_{2}\right)=\varnothing$.

\section{Monotonicity AND PACKING Numbers}

In this section we describe a monotone formula which plays an important role in the constructions of subsequent sections. 
Definition 3.1 (Packing). Let $X$ be a metric space and $S \subseteq X$ with $\operatorname{diam}(S)<\infty$. For $\epsilon>0$, we say that a subset $\mathbf{x} \equiv\left\{x_{i}\right\} \subseteq S$ is an $\epsilon$-subpacking if

$$
d\left(x_{i}, x_{j}\right) \geq \epsilon \operatorname{diam}(S) \text { for every } i \neq j .
$$

An $\epsilon$-subpacking $\mathbf{x}$ is said to be a packing if it is also $\epsilon \operatorname{diam}(S)$-dense in $S$.

We write $|\mathbf{x}|=N$ as the number of elements in $\mathbf{x}$ if it is finite. If we want to signify the set in question we may write $\mathbf{x}=\mathbf{x}(S)$. We define the $\epsilon$-packing number $P_{\epsilon}(S)$ by

$$
P_{\epsilon}(S) \equiv \sup \{|\mathbf{x}|: \mathbf{x} \text { is an } \epsilon \text {-subpacking for } S\} .
$$

A packing $\mathbf{x}$ is called a maximal $\epsilon$-packing of $S$ if $|\mathbf{x}|=P_{\epsilon}(S)<\infty$.

In the case that $S=B_{r}(p)$ is a metric ball we may write $\mathbf{x}(p, r)=\mathbf{x}\left(B_{r}(p)\right)$ and the $\epsilon$-packing number $P_{\epsilon}(p, r) \equiv P_{\epsilon}\left(B_{r}(p)\right)$. Let us record some easy but useful properties which hold for general metric spaces.

Lemma 3.2. Let $X$ be a metric space with $\epsilon>0$ fixed. Then the following hold:

(i) (Enlargement) If $\mathbf{x}$ is an $\epsilon$-subpacking of $B_{r}(x)$, then either $\mathbf{x}$ is an $\epsilon$-packing or there exists $x^{\prime} \in B_{r}(x)$ such that $\mathbf{x}^{\prime} \equiv \mathbf{x} \cup\left\{x^{\prime}\right\}$ is also an $\epsilon$-subpacking.

(ii) (Maximal subpacking $\Longrightarrow$ packing) If $\mathbf{x}$ is an $\epsilon$-subpacking of $B_{r}(x)$ with $|\mathbf{x}|=P_{\epsilon}(x, r)<\infty$, then $\mathbf{x}$ is an $\epsilon$-packing.

(iii) ( $\epsilon$-monotonicity) If $\mathbf{x}$ is an $\epsilon$-subpacking and $\epsilon^{\prime}<\epsilon$, then $\mathbf{x}$ is an $\epsilon^{\prime}$-subpacking. In particular, for each $r>0$ we have that $P_{\epsilon^{\prime}}(x, r) \geq P_{\epsilon}(x, r)$.

We wish to now discuss some more refined properties of $\epsilon$-packings and packing numbers for Alexandrov spaces. To do this let us introduce the induced subpacking. Indeed, this notation makes sense for any locally compact length metric space, but it is not so useful in general.

Definition 3.3 (Induced subpacking). Let $p \in X, R>0$ and for each $x \in \bar{B}_{R}(p) \backslash\{p\}$ we fix a geodesic $\gamma_{p x}=\gamma_{p x}^{R}$ connecting $p$ and $x$. Given $0<r<R$, we define the inducting function $\varphi_{r}^{R}: \bar{B}_{R}(p) \rightarrow \bar{B}_{r}(p)$, $x \mapsto \bar{x}$, where $\bar{x} \in \gamma_{p x}^{R}$ is the point with $d(p, \bar{x})=\frac{r}{R} \cdot d(p, x)$. Now let $\left\{x_{i}\right\}_{i=1}^{N}$ be an $\epsilon$-subpacking of $\bar{B}_{R}(p)$ and $0<r<R$, then we call the collection of points $\left\{\varphi_{r}^{R}\left(x_{i}\right)\right\}_{i=1}^{N}$ the induced subpacking in $\bar{B}_{r}(p)$ of $\left\{x_{i}\right\}_{i=1}^{N}$.

Note that the choice of geodesic $\gamma_{p x}$ in the definition of $\varphi_{r}^{R}$ is certainly not unique. However in the above definition of $\varphi_{r}^{R}$, such a choice is fixed for a given $R>0$ while independent of $0<r \leq R$. If no confusion arises one may write $\gamma_{p x}^{R}=\gamma_{p x}$.

The proof of the following propositions are easy exercises based on the Toponogov comparisons.

Proposition 3.4. Let $0<\epsilon, R<1$. The following hold for any $(X, p) \in$ Alex $^{n}(-\epsilon)$ with $1 \geq c \geq 1-R^{2}$. If $(X, p) \in$ Alex $^{n}(0)$, then $c \equiv 1$ can be chosen as a constant.

(i) (Induced Packing) Let $\left\{x_{i}\right\}$ be an $\epsilon$-subpacking of $B_{R}(p)$. For any $0<r<R$, the induced subpacking in $B_{r}(p)$ is a ce-subpacking.

(ii) (Monotonicity) If $r \leq R$, then the packing number $P_{c \epsilon}(x, r) \geq P_{\epsilon}(x, R)$. 
(iii) (Bounds) If $\mathbf{x}=\left\{x_{i}\right\}$ is an $\epsilon$-subpacking for $B_{r}(p)$ with $0<r \leq 1$, then $1 \leq|\mathbf{x}| \leq C(n) \epsilon^{-n}$. In particular, we have $1 \leq P_{\epsilon}(p, r) \leq C(n) \epsilon^{-n}$.

(iv) (Density) There exists a limit $\lim _{r \rightarrow 0} P_{\epsilon}(p, r) \equiv P_{\epsilon}(p) \leq C(n) \epsilon^{-n}$, which we call the $\epsilon$-density at $x$. In fact, $P_{\epsilon}(p)=P_{\epsilon}\left(p^{*}, 1\right)$, where $p^{*}$ is the cone point in the tangent cone at $p$.

\section{BAD SCALES}

This section is dedicated to proving Lemma 2.2. It says that there are at most a finite number of bad scales at each point, and our space has a fixed cone structure which persists over all good scales. Let us begin with an easy proposition.

Proposition 4.1. For any $0 \leq r<R / 2<R \leq 1$, if $(r, R) \cap \operatorname{Bad}^{\epsilon}(x)=\varnothing$, then $T_{x}^{\epsilon}(r, R)=0$.

Proof. The proof is almost taulogical. Let $r_{\beta_{(k)}}=\inf \left\{r_{\beta} \in \operatorname{Bad}^{\epsilon}(x): r_{\beta} \geq R\right\}$ and $r_{\beta(k+1)}$ be the next $\epsilon$-bad scale. Because $(r, R) \cap \operatorname{Bad}^{\epsilon}(x)=\varnothing$ we have that $r_{\beta_{(k+1)}} \leq r<R / 2<R \leq r_{\beta_{(k)}}$. Therefore, $r_{\beta_{(k)}} / r_{\beta_{(k+1)}}>2$ and $\beta_{(k+1)}-\beta_{(k)} \geq 2$. By the definition $T_{x}^{\epsilon}\left(r_{\beta_{(k+1)}}, r_{\beta_{(k)}}\right)=0$. Note that $[r, R] \subseteq\left[r_{\beta_{(k+1)}}, r_{\beta_{(k)}}\right]$ and so we have that $T_{x}^{\epsilon}(r, R)=0$.

To prove Lemma 2.2, we need a result of the form "almost packing cone implies almost metric cone". We begin with the following proposition. It follows directly from the definitions of $\epsilon$-packing and Hausdorff distance.

Proposition 4.2. Let $X$ and $Y$ be metric spaces whose diameters are both no more than 1. Let $\left\{x_{i}\right\}_{i=1}^{N_{1}}$ be an $\epsilon$-packing of $X$ and $\left\{y_{i}\right\}_{i=1}^{N_{2}}$ be an $\epsilon$-packing of $Y$. If $N_{1}=N_{2}=N$ and

$$
\left|d\left(x_{i}, x_{j}\right)-d\left(y_{i}, y_{j}\right)\right| \leq \epsilon
$$

for every $1 \leq i, j \leq N$, then $d_{G H}(X, Y) \leq 4 \epsilon$.

The first main result of this section is the following:

Lemma 4.3 (Almost packing cone implies almost metric cone). There is a universal constant $c>0$ such that the following holds for any $n \in \mathbb{N}$ and $\epsilon \in(0, c)$. Let $(X, p) \in \operatorname{Alex}^{n}(-\epsilon)$ and $0 \leq r \leq \frac{1}{2} R \leq c$. Let $\mathbf{x}(p, R)=\left\{x_{i}\right\}_{i=1}^{N}$ be a $\epsilon$-packing of $B_{R}(p)$. We have

$$
T_{p}^{\epsilon^{0.1}}(r, R)=0
$$

if both of the following are satisfied.

(i) $P_{\epsilon}(p, r)=N=P_{\epsilon}(p, R)$.

(ii) $r^{-1} d\left(\varphi_{r}^{R}\left(x_{i}\right), \varphi_{r}^{R}\left(x_{j}\right)\right) \leq R^{-1} d\left(x_{i}, x_{j}\right)+\epsilon$, for every $1 \leq i, j \leq N$.

Here $\varphi_{r}^{R}: \bar{B}_{R}(p) \rightarrow \bar{B}_{r}(p)$ is the inducing function defined as in Definition 3.3. 
Proof of Lemma 4.3. Let us introduce the notation $(\lambda) \bar{B}_{s} \equiv\left(\bar{B}_{s}, \lambda d\right)$ to denote the rescaled space. The proof consists of two points. First, we will see that it is almost immediate from the assumed conditions that the mapping $\varphi_{r}^{R}: \bar{B}_{R}(p) \rightarrow(R / s) \bar{B}_{s}(p)$ is a GH map. Second, we will show that $\bar{B}_{R}(p)$ is GH-close to a ball in a cone space $C(\Sigma)$, centered at the cone point. The combination of these two points prove the Lemma.

Let us discuss these points more carefully. For simplicity, we will only prove the result for $X \in \operatorname{Alex}^{n}(0)$. The general case is similar with a modifications on $c$, which are just used to estimate the law of cosine formula in (4.17). Now by the assumptions and the monotonic property, the induced subpacking $\left\{\varphi_{s}^{R}\left(x_{i}\right)\right.$ : $\left.x_{i} \in \mathbf{x}(p, R)\right\}$ is a packing of $\bar{B}_{s}(p)$ and

$$
R^{-1} d\left(x_{i}, x_{j}\right) \leq s^{-1} d\left(\varphi_{s}^{R}\left(x_{i}\right), \varphi_{s}^{R}\left(x_{j}\right)\right) \leq R^{-1} d\left(x_{i}, x_{j}\right)+\epsilon,
$$

for every $s \in[r, R)$ and every $1 \leq i \neq j \leq N$.

By Proposition 4.2, for all $s \in[r, R)$, we therefore have that

$$
d_{G H}\left(\bar{B}_{R}(p),(R / s) \bar{B}_{s}(p)\right) \leq 4 \epsilon R,
$$

where $\varphi_{s}^{R}: \bar{B}_{R}(p) \rightarrow(R / s) \bar{B}_{s}(p)$ is a $8 \epsilon R$-isometry. To prove (4.1) it therefore suffices to construct a metric cone $C(\Sigma)$ and show that

$$
d_{G H}\left(\bar{B}_{R}(p), \bar{B}_{R}\left(p^{*}\right)\right) \leq \frac{1}{2} \epsilon^{0.1} R,
$$

where $p^{*} \in C(\Sigma)$ is the cone point. Let us prove this by first assuming the following lemma, which we will prove later. Let $\iota_{\lambda}: \bar{B}_{s} \rightarrow(\lambda) \bar{B}_{s}$ be the identity map.

Lemma 4.4. Let $S_{\rho}=\left\{x \in \bar{B}_{R}(p): d(p, x)=\rho\right\}$ be the $\rho$-cross section in $X$.

(i) For any $t \in\left[\epsilon^{0.5}, 1\right)$, the restricted map $\left.\iota_{t^{-1}} \circ \varphi_{t R}^{R}\right|_{S_{R}}: S_{R} \rightarrow\left(t^{-1}\right) S_{t R}$ is $\epsilon^{0.4} R$-onto.

(ii) For every $x, y \in S_{R}$ and $t_{1}, t_{2} \in\left[\epsilon^{0.5}, 1\right)$, we have

$$
\left|\cos \tilde{\measuredangle}\left(p_{y}^{x}\right)-\cos \tilde{\measuredangle}\left(p_{\varphi_{t_{2} R}^{R}(y)}^{\varphi_{t_{1}}^{R}(x)}\right)\right| \leq \epsilon^{0.4} .
$$

(iii) For any $x, y \in S_{R}$, geodesic triangle $\triangle$ pxy is $\epsilon^{0.3} R$-close to a geodesic triangle in $\mathbb{R}^{2}$, equipped with the extrinsic metrics.

Using the above we now construct a metric cone $C(\Sigma)$ and define a GH-map $f: \bar{B}_{R}\left(p^{*}\right) \rightarrow \bar{B}_{R}(p)$, where $p^{*} \in C(\Sigma)$ is the cone point. Define a distance function $d_{S_{R}}$ on the $R$-cross section $S_{R}=\{x \in X: d(p, x)=R\}$ by

$$
d_{S_{R}}(x, y)=\inf _{x_{0}, \ldots, x_{N} \in S_{R}}\left\{\sum_{\alpha=1}^{N} d_{X}\left(w_{\alpha-1}, w_{\alpha}\right): w_{0}=x, w_{N}=y, d_{X}\left(w_{\alpha-1}, w_{\alpha}\right) \leq \epsilon^{0.1} R\right\} .
$$

Note that this is an approximation of the induced length space distance function on a subset. It's clear that $d_{S_{R}}(x, y) \geq d_{X}(x, y)$, and thus if $d_{S_{R}}(x, y)=0$ then $x=y$. To verify the triangle inequality, we let $x, y, z \in S_{R}$. By the definition we have for any $\eta>0$ that there exists $w_{\alpha} \in S_{R}$ with $w_{0}=x, w_{N_{1}}=y, w_{N_{2}}=z$, 
$d_{X}\left(w_{\alpha-1}, w_{\alpha}\right) \leq \epsilon^{0.1} R$, so that $d_{S_{R}}(x, y) \geq \sum_{\alpha=1}^{N_{1}} d_{X}\left(w_{\alpha-1}, w_{\alpha}\right)-\eta$ and $d_{S_{R}}(y, z) \geq \sum_{\alpha=N_{1}+1}^{N_{2}} d_{X}\left(w_{\alpha-1}, w_{\alpha}\right)-\eta$. Then we have

$$
d_{S_{R}}(x, y)+d_{S_{R}}(y, z) \geq \sum_{i=1}^{N_{2}} d_{X}\left(w_{\alpha-1}, w_{\alpha}\right)-2 \eta \geq d_{S_{R}}(x, z)-2 \eta .
$$

Letting $\eta \rightarrow 0$ we then obtain the triangle inequality.

Now let $\left(\Sigma, d_{\Sigma}\right)=\left(S_{R}, \frac{1}{R} d_{S_{R}}\right)$ and $C(\Sigma)$ be the metric cone over $\Sigma$ and $p^{*}$ is the cone point. Let $\left(\tilde{S}_{R}, d_{\tilde{S}_{R}}\right)=$ $\left(\Sigma, R d_{\Sigma}\right)$ be the $R$-cross section in $C(\Sigma)$. Let $\Pi: C(\Sigma) \rightarrow \tilde{S}_{R}$ by $a=\left(\bar{a}, d\left(p^{*}, a\right)\right) \mapsto \bar{a}$ be the projection mapping. Identify $\tilde{S}_{R}$ with $S_{R}$ and let us define

$$
f: \bar{B}_{R}\left(p^{*}\right) \rightarrow \bar{B}_{R}(p) \text { by } a \mapsto \varphi_{d\left(p^{*}, a\right)}^{R} \circ \Pi(a) .
$$

We first show that $f$ is $\epsilon^{0.4} R$-onto. Let $x \in \bar{B}_{R}(p)$. Note that for any $y \in \tilde{S}_{R}=S_{R}$, we have $\left(y, d_{X}(p, x)\right) \in$ $C(\Sigma)$ and $f\left(\left(y, d_{X}(p, x)\right)\right)=\varphi_{d(p, x)}^{R}(y)$. Thus the $\epsilon^{0.4} R$-onto property of $f$ follows from Lemma 4.4 (i).

Now we show that $f$ is $\frac{1}{2} \epsilon^{0.1} R$-distance preserving. Let $a, b \in C(\Sigma), x=f(a), y=f(b)$ and $\gamma_{x, y}$ be a geodesic connecting $x$ and $y$. By Lemma 4.4 (i), for any partition $\left\{u_{i}\right\}$ of $\gamma_{x, y}$, there exist $w_{i} \in S_{R}$ and $s_{i}>0$ such that $d_{X}\left(\varphi_{s_{i} R}^{R}\left(w_{i}\right), u_{i}\right) \leq \epsilon^{0.4} R$. Note that for any two points $x^{\prime}, y^{\prime} \in S_{R}$ we have that $d_{S_{R}}\left(x^{\prime}, y^{\prime}\right)=d_{X}\left(x^{\prime}, y^{\prime}\right)$ if $d_{X}\left(x^{\prime}, y^{\prime}\right) \leq \epsilon^{0.1} R$. By Lemma 4.4 (iii), the points $u_{i}$ and $w_{i}, i=1, \ldots, N$ can be chosen so that $\frac{1}{2} \epsilon^{0.1} R \geq d_{X}\left(w_{i-1}, w_{i}\right)=d_{S_{R}}\left(w_{i-1}, w_{i}\right) \geq \frac{1}{4} \epsilon^{0.1} R$. Thus for this partition we have that $N \leq \frac{10 R}{\epsilon^{0.1} R}=10 \epsilon^{-0.1}$.

Now let $\tilde{\varphi}$ be the inducting function on $C(\Sigma)$ defined in the same way as $\varphi$. By the cone metric, we have

$$
d_{C(\Sigma)}\left(\tilde{\varphi}_{s_{i-1} R}^{R}\left(w_{i-1}\right), \tilde{\varphi}_{s_{i} R}^{R}\left(w_{i}\right)\right)=\sqrt{s_{i-1} s_{i} d_{S_{R}}^{2}\left(w_{i-1}, w_{i}\right)+\left(s_{i-1}-s_{i}\right)^{2} R^{2}} .
$$

By Lemma 4.4 (iii), we have

$$
\left|d_{X}\left(\varphi_{s_{i-1} R}^{R}\left(w_{i-1}\right), \varphi_{s_{i} R}^{R}\left(w_{i}\right)\right)-\sqrt{s_{i-1} s_{i} d_{X}^{2}\left(w_{i-1}, w_{i}\right)+\left(s_{i-1}-s_{i}\right)^{2} R^{2}}\right|<10 \epsilon^{0.3} R .
$$

Therefore,

$$
\begin{aligned}
d_{X}(x, y) & =\sum_{i} d_{X}\left(u_{i-1}, u_{i}\right) \\
& \geq \sum_{i=1}^{N}\left(d_{X}\left(\varphi_{s_{i-1} R}^{R}\left(w_{i-1}\right), \varphi_{s_{i} R}^{R}\left(w_{i}\right)\right)-2 \epsilon^{0.4} R\right) \\
& \geq-12 N \cdot \epsilon^{0.3} R+\sum_{i=1}^{N} \sqrt{s_{i-1} s_{i} d_{X}^{2}\left(w_{i-1}, w_{i}\right)+\left(s_{i-1}-s_{i}\right)^{2} R^{2}} \\
& \geq-120 \epsilon^{0.2} R+\sum_{i=1}^{N} \sqrt{s_{i-1} s_{i} d_{S_{R}}^{2}\left(w_{i-1}, w_{i}\right)+\left(s_{i-1}-s_{i}\right)^{2} R^{2}} \\
& =-120 \epsilon^{0.2} R+\sum_{i=1}^{N} d_{C(\Sigma)}\left(\tilde{\varphi}_{s_{i-1} R}^{R}\left(w_{i-1}\right), \tilde{\varphi}_{s_{i} R}^{R}\left(w_{i}\right)\right) \\
& \geq-122 \epsilon^{0.2} R+d_{C(\Sigma)}(a, b) .
\end{aligned}
$$


The last inequality follows from the triangle inequality since $w_{1}$ and $w_{N}$ can be chosen so that $d\left(a, \tilde{\varphi}_{s_{1} R}^{R}\left(w_{1}\right)\right)<$ $\epsilon^{0.4} R$ and $d\left(b, \tilde{\varphi}_{s_{N} R}^{R}\left(w_{N}\right)\right)<\epsilon^{0.4} R$. Starting from a partition of $\gamma_{a, b}$ and apply the same arguments. We get

$$
d_{X}(x, y) \leq d_{C(\Sigma)}(a, b)+122 \epsilon^{0.2} R .
$$

Combining (4.11), (4.12) and the definition of $f$, we get the desired result.

Now let us finish the proof of Lemma 4.4:

Proof of Lemma 4.4. (i) By (4.3), for any $\lambda \in[r / R, 1) \supseteq[1 / 2,1)$ we have that

$$
\left.\iota_{\lambda^{-1}} \circ \varphi_{\lambda R}^{R}\right|_{\rho}: S_{\rho} \rightarrow\left(\lambda^{-1}\right) S_{\lambda \rho} \text { is a } 24 \epsilon R \text {-isometry for any } \rho \in(0, R]
$$

This in particular proves (i) for $t \in[r / R, 1)$. For the case $t \ll r / R$, we need to inductively apply $\varphi_{R / 2}^{R}$.

For any $t \in\left[\epsilon^{0.5}, 1\right)$, there is an integer $K=K(t) \leq \epsilon^{-0.1}$ such that $2^{-(K+1)} \leq t<2^{-K}$. Since in $X$ geodesics do not bifurcate and in the definition of induced packing, the choices of geodesics are a priori fixed in terms of $p$ and $R$, we can write

$$
\varphi_{t R}^{R}=\varphi_{2^{K} t R}^{R} \circ \underbrace{\varphi_{R / 2}^{R} \circ \cdots \circ \varphi_{R / 2}^{R}}_{K} .
$$

Note that $2^{K} t \in[1 / 2,1) \subseteq[r / R, 1)$. Thus (4.13) applies to $\rho=R$ and $\lambda=2^{K} t$. Combining (4.13) and (4.14), we get that $\iota_{t^{-1}} \circ \varphi_{t R}^{R}$ is $24(K+1) \epsilon R$-onto. Then the result follows since $K \leq \epsilon^{-0.1}$.

(ii) We first show that (4.5) is true for $t_{1}=t_{2}=t$. Fix $t \in\left[\epsilon^{0.5}, 1\right)$. Let $x_{0}=x, x_{i}=\varphi_{R / 2}^{R}\left(x_{i-1}\right)$ for $1 \leq i \leq K$ and $x_{K+1}=\varphi_{2^{K} t R}^{R}\left(x_{K}\right)=\varphi_{t R}^{R}(x)$, where $K=K(t) \leq \epsilon^{-0.1}$ is defined as in (i). The sequence $\left\{y_{i}\right\}$ is defined similarly in terms of $y$. By (4.13) and because $2^{K} t \in[1 / 2,1)$, for $1 \leq i \leq K$ we have

$$
\left|\frac{1}{2} d\left(x_{i}, y_{i}\right)-d\left(x_{i-1}, y_{i-1}\right)\right| \leq 24 \epsilon R
$$

and

$$
\left|2^{K} t \cdot d\left(x_{K+1}, y_{K+1}\right)-d\left(x_{K}, y_{K}\right)\right| \leq 24 \epsilon R .
$$

Note that $d\left(p, x_{i-1}\right)=d\left(p, y_{i-1}\right)=\frac{1}{2} d\left(p, x_{i}\right)=\frac{1}{2} d\left(p, y_{i}\right)$ and $d\left(p, x_{K}\right)=d\left(p, y_{K}\right)=2^{K} t \cdot d\left(p, x_{K+1}\right)=$ $2^{K} t \cdot d\left(p, y_{K+1}\right)$. By law of cosine, we have

$$
\begin{aligned}
\left|\cos \tilde{\measuredangle}\left(p_{y_{i}}^{x_{i}}\right)-\cos \tilde{\measuredangle}\left(p_{y_{i-1}}^{x_{i-1}}\right)\right| & =\frac{1}{2}\left|\left(\frac{d\left(x_{i}, y_{i}\right)}{d\left(p, x_{i}\right)}\right)^{2}-\left(\frac{d\left(x_{i-1}, y_{i-1}\right)}{d\left(p, x_{i-1}\right)}\right)^{2}\right| \\
& \leq \frac{50 \epsilon R}{d\left(p, x_{i}\right)} \leq \frac{50 \epsilon R}{t R} \\
& \leq 50 \epsilon^{0.5} .
\end{aligned}
$$

Summing up (4.17) for $i=1,2, \ldots, K+1$, we get

$$
\left|\cos \tilde{\measuredangle}\left(p_{y}^{x}\right)-\cos \tilde{\measuredangle}\left(p_{\varphi_{t R}^{R}(y)}^{\varphi_{t R}^{R}(x)}\right)\right| \leq 50(K+1) \epsilon^{0.5} \leq \epsilon^{0.4} .
$$


Suppose $t_{1} \leq t_{2}$. By Topnogov comparison, we have

$$
\tilde{\measuredangle}\left(p_{y}^{x}\right) \leq \tilde{\measuredangle}\left(p_{\varphi_{t_{2} R}^{R}(y)}^{R}(x) \leq \tilde{\measuredangle}\left(p_{\varphi_{t_{1} R}^{R}(x)}^{R}\right)\right.
$$

Then (4.5) follows from (4.18) and (4.19).

The statement (iii) is a direct consequence of (ii).

Lemma 4.3 implies that when passing an $\epsilon$-bad scale, either the packing number, or the rescaled distance distortion is increased by at least a definite amount, depending on $\epsilon$.

Corollary 4.5. For any $\epsilon>0$, there is $\delta=\delta(\epsilon)>0$ such that the following holds. Let $(X, p) \in$ Alex $^{n}(-\delta)$ be an Alexandrov space with $r_{\beta_{(k)}}, r_{\beta_{(k+1)}} \epsilon$-bad scales of $p$. Let $\left\{x_{i}\right\}$ be a maximal $\delta$-packing of $B_{r_{\beta(k)}}(p)$ and $\left\{y_{i}\right\}$ be the induced subpacking in $B_{r_{(k+1)+1}}(p)$. Then one of the following holds:

(i) $P_{\delta}\left(p, r_{\beta_{(k+1)}+1}\right) \geq P_{\delta}\left(p, r_{\beta_{(k)}}\right)+1$;

(ii) there exist $i \neq j$ such that $r_{\beta_{(k+1)}+1}^{-1} d\left(y_{i}, y_{j}\right)>r_{\beta_{(k)}}^{-1} d\left(x_{i}, x_{j}\right)+\delta$.

Proof. By the definition of bad scales, we have that $T_{p}^{\epsilon}\left(r_{\beta_{(k+1)}+1}, r_{\beta_{(k)}}\right)=1$. Then the result follows from Lemma 4.3 with $\delta=\epsilon^{10}$.

Now we give a proof of Lemma 2.2 using the above monotone property.

Proof of Lemma 2.2. We will only prove for $X \in \mathrm{Alex}^{n}(0)$ to keep notation simple, the general case is similar. Let $r_{\alpha}=2^{-\alpha}, \alpha \in \mathbb{N}$ and $K>J \geq 0$ be integers. Let $N_{J}=P_{\delta}\left(p, r_{J}\right)$ be the maximum $\delta$-packing number of $B_{r_{J}}(p)$. Let $\mathbb{I}=\left\{\beta_{(k)} \in \mathbb{N}: r_{\beta_{(k)}} \in \operatorname{Bad}^{\epsilon}(p) \cap\left[r_{K}, r_{J}\right]\right\}$. We claim that if $|\mathbb{I}|>10 N_{J}^{2} \delta^{-1}$, then $P_{\delta}\left(p, r_{K}\right) \geq P_{\delta}\left(p, r_{J}\right)+1$. If this is not true, then $P_{\delta}\left(p, r_{K}\right)=P_{\delta}\left(p, r_{\alpha}\right)=P_{\delta}\left(p, r_{J}\right)$ for every $\alpha \in[J, K] \cap \mathbb{Z}$. Let $\left\{x_{i}\right\}_{i=1}^{N_{J}}$ be a maximal $\delta$-packing of $B_{r_{J}}(p)$ and $\left\{x_{i}^{\alpha}\right\}$ be the induced subpacking in $B_{r_{\alpha}}(p)$. By Corollary 4.5 , for every $\beta_{(k)} \in \mathbb{I}$, there exist $i$ and $j$, depending on $\beta_{(k)}$, such that

$$
r_{\beta_{(k+1)}+1}^{-1} d\left(x_{i}^{\beta_{(k+1)}+1}, x_{j}^{\beta_{(k+1)}+1}\right)>r_{\beta_{(k)}}^{-1} d\left(x_{i}^{\beta_{(k)}}, x_{j}^{\beta_{(k)}}\right)+\delta .
$$

Given a pair of indices $(i, j)$, let $\mathbb{I}_{(i, j)}$ be the collection of $\beta_{(k)} \in \mathbb{I}$ such that (4.20) holds. Because $|\mathbb{I}|>10 N_{J}^{2} \delta^{-1}$, there exist $1 \leq i_{0}, j_{0} \leq N_{J}$, such that $\left|\mathbb{I}_{\left(i_{0}, j_{0}\right)}\right|>10 \delta^{-1}$. Furthermore, there is a subset $\mathbb{J}_{\left(i_{0}, j_{0}\right)} \subseteq \mathbb{I}_{\left(i_{0}, j_{0}\right)}$ with $\left|\mathbb{J}_{\left(i_{0}, j_{0}\right)}\right|>5 \delta^{-1}$, so that the intervals $\left\{\left(\beta_{(k)}, \beta_{(k+1)}+1\right): \beta_{(k)} \in \mathbb{J}_{\left(i_{0}, j_{0}\right)}\right\}$ are disjoint. Note that by the monotonic property, we have

$$
r_{\alpha_{1}}^{-1} d\left(x_{i}^{\alpha_{1}}, x_{j}^{\alpha_{1}}\right) \geq r_{\alpha_{2}}^{-1} d\left(x_{i}^{\alpha_{2}}, x_{j}^{\alpha_{2}}\right)
$$

for every $\alpha_{1} \geq \alpha_{2}$. Summing up (4.20) for $\beta_{(k)} \in \mathbb{J}_{\left(i_{0}, j_{0}\right)}$ and taking in account (4.21), we get

$$
2 \geq r_{K}^{-1} d\left(x_{i}^{K}, x_{j}^{K}\right)>r_{J}^{-1} d\left(x_{i}^{J}, x_{j}^{J}\right)+\sum_{\beta(k) \in \mathbb{J}_{\left(i_{0}, j_{0}\right)}} \delta \geq 5,
$$

a contradiction. 
Note now that for every $r>0$ we have that $P_{\delta}(p, r) \leq C(n, \delta)$. Thus it follows from the above claim that

$$
\left|\operatorname{Bad}^{\epsilon}(p)\right| \leq(C(n, \delta)+1)\left(10 C(n, \delta)^{2} \delta^{-1}+1\right) .
$$

For any $\lambda \in(0,1 / 4)$, it follows from Lemma 2.2 that for any $x \in X$, there is at least one of the intervals

$$
\left[\lambda^{2 \operatorname{Bad}^{\epsilon}(x)+1}, \lambda^{2 \operatorname{Bad}^{\epsilon}(x)}\right], \ldots,\left[\lambda^{5}, \lambda^{4}\right],\left[\lambda^{3}, \lambda^{2}\right],[\lambda, 1]
$$

containing no $\epsilon$-bad scale. Thus we have

Lemma 4.6. For any $n \in \mathbb{N}, 1 / 4>\lambda>0$ and $\delta>0$, there exists $\eta=\eta(n, \delta, \lambda)>0$ such that for any $x \in X \in \operatorname{Alex}^{n}(-1)$ and any $0<R \leq 1$, there exists $r_{x} \geq \eta R$, such that $\operatorname{Bad}^{\delta}(x) \cap\left[\lambda r_{x}, r_{x}\right]=\varnothing$ and thus $T_{x}^{\delta}\left(\lambda r_{x}, r_{x}\right)=0$.

\section{Splitting TheORY AND Dimension Reduction}

5.1. Splitting theory. In this subsection, we discuss the splitting theory in Alexandrov geometry. Proposition 5.1 is a key geometric property for spaces with lower sectional curvature bounds that distinguishes them from spaces with lesser geometric constraints, such as lower Ricci curvature bounds. In words, it says that if some ball almost-splits off a Euclidean factor, then all sub-balls continue to almost-split off this factor.

Proposition 5.1. For any $n, \epsilon>0$, there exist $\delta=\delta(n, \epsilon)>0$ so that the following holds for any $X \in$ $\operatorname{Alex}^{n}(-\delta)$ and $R \in(0,1]$.

(i) Let $u=\left(u_{1}, \ldots, u_{k}\right): B_{5 R}(p) \rightarrow \mathbb{R}^{k}$ be $a(k, \delta)$-splitting map. For any $B_{r} \subseteq B_{R}(p)$ and any $\xi \in u\left(B_{r}\right)$, there exists a map $\phi: B_{r} \rightarrow u^{-1}(\xi)$ so that

$$
(u, \phi): B_{r} \rightarrow \mathbb{R}^{k} \times u^{-1}(\xi)
$$

is er-isometry.

(ii) If $f: B_{5 R}(p) \rightarrow B_{5 R}(z)$, where $z \in \mathbb{R}^{k} \times Z$, is a $\delta R$-isometry, then there exists a $(k, \epsilon)$-splitting map $u: B_{R}(p) \rightarrow \mathbb{R}^{k}$.

(iii) If there is a $(k, \delta)$-strainer $\left\{\left(a_{i}, b_{i}\right)\right\}$ with $d\left(p, a_{i}\right), d\left(p, b_{i}\right) \geq 5 R$ for every $1 \leq i \leq k$, then there exists $a(k, \epsilon)$-splitting map $u: B_{R}(p) \rightarrow \mathbb{R}^{k}$.

The above splitting theory in Alexandrov geometry is well understood. For completeness we outline the proof.

Proof. We argue $(i)$ by contradiction. This argument can be made effective with some extra work. Note that if $X_{i} \in \operatorname{Alex}^{n}(\kappa)$ with $X_{i} \rightarrow X$ and $\delta_{i} \rightarrow \delta$, then the limit of $\left(k, \delta_{i}\right)$-splitting functions on $X_{i}$ is a $(k, \delta)$-splitting function on $X$. Thus passing to a limit of contradictive rescalled sub-balls, it suffices to show 
that if $(X, p) \in \operatorname{Alex}^{n}(0)$ and there is a 0 -splitting function $u=\left(u_{1}, \ldots, u_{k}\right): B_{5}(p) \rightarrow \mathbb{R}^{k}$, then for any $\xi \in u\left(B_{1}(p)\right)$, there exists a map $\phi: B_{1}(p) \rightarrow u^{-1}(\xi)$ so that

$$
(u, \phi): B_{1}(p) \rightarrow \mathbb{R}^{k} \times u^{-1}(\xi)
$$

is an isometric embedding. For such a 0 -splitting function $u$, the following hold for every $i$ and $j$ :

(1) $u_{i}$ is 0 -concave.

(2) $\left\langle\nabla u_{i}, \nabla u_{j}\right\rangle=\delta_{i j}$.

(3) For any $x, y \in B_{R}(p)$ and any minimizing geodesic $\gamma$ connecting $x$ and $y$, it holds that

$$
\left\langle\uparrow_{x}^{y}, \nabla_{x} u_{i}\right\rangle+\left\langle\uparrow_{y}^{x}, \nabla_{y} u_{i}\right\rangle=0
$$

We now prove the result by induction on $k$. Start with the base case $k=1$. Let $\sigma_{x}(t)$ be a $u$-gradient flow with $\sigma_{x}(0)=x$. If no confusion arises one may write $x_{t}=\sigma_{x}(t)$. Because $u$ is 0 -concave and $|\nabla u|=1$, we have $u\left(x_{t}\right)-u(x)=t$ and $d\left(u\left(x_{t}\right), u\left(x_{s}\right)\right)=|t-s|$. In particular, $\sigma_{x}(t)$ is a geodesic from $x$. It's clear that the directed tangent vectors $\sigma^{+}(t)=\nabla_{x_{t}} u$ and $\sigma^{-}(t)=-\nabla_{x_{t}} u$.

Let $T_{x}$ be the time so that $\sigma_{x}\left(T_{x}\right) \in u^{-1}(\xi)$ and define $\phi(x)=\sigma_{x}\left(T_{x}\right) \in u^{-1}(\xi)$. We will show that

$$
\left.(u, \phi)\right|_{B_{1}}: B_{1} \rightarrow \mathbb{R} \times u^{-1}(\xi)
$$

is an isometric embedding. This follows from the following statements for arbitrary $\xi \in u\left(B_{1}(p)\right)$ and $t, s \in[0,1]$.

(A) $\left|T_{x}\right|=d\left(x, u^{-1}(\xi)\right)$.

(B) For any two $u$-gradient curves $\alpha$ and $\beta$, we have $d(\alpha(t), \beta(t))=d(\alpha(s), \beta(s))$.

(C) The Pythagorean Theorem $d^{2}\left(x_{t}, y\right)=d^{2}(x, y)+t^{2}$.

We first prove (A). It's clear that $\left|T_{x}\right| \geq d\left(x, u^{-1}(\xi)\right)$. Recall that if $X \in \operatorname{Alex}^{n}(0)$ and $f: X \rightarrow \mathbb{R}$ is a $\lambda$-concave function, then

$$
d(p, q) \cdot\left\langle\uparrow_{p}^{q}, \nabla_{p} f\right\rangle \geq f(q)-f(p)-\frac{\lambda}{2} \cdot d^{2}(p, q) .
$$

Thus if $u: X \rightarrow \mathbb{R}$ is a 0 -concave function, then

$$
d(p, q) \geq d(p, q) \cdot\left\langle\uparrow_{p}^{q}, \nabla_{p} u\right\rangle \geq u(q)-u(p) .
$$

Let $y \in u^{-1}(\xi)$ so that $d(x, y)=d\left(x, u^{-1}(\xi)\right)$, then we have

$$
d(x, y) \geq|u(x)-u(y)|=\left|u\left(\sigma_{x}(0)\right)-\xi\right|=\left|u\left(\sigma_{x}(0)\right)-u\left(\sigma_{x}\left(T_{x}\right)\right)\right|=\left|T_{x}\right| .
$$

To prove (B), we let $x_{t}=\alpha(t), y_{t}=\beta(t)$ and $\ell(t)=d\left(x_{t}, y_{t}\right)$. Assume $t \geq s$. Let $\ell^{+}(t)=\lim _{\eta \rightarrow 0^{+}} \frac{\ell(t+\eta)-\ell(t)}{\eta}$ and $\ell^{-}(t)=\lim _{\eta \rightarrow 0^{+}} \frac{\ell(t-\eta)-\ell(t)}{\eta}$ be the one-sided derivatives. By the first variation formula and because $u$ is 0 -concave, we have

$$
\ell^{+}(t) \leq-\left\langle\uparrow_{x_{t}}^{y_{t}}, \nabla_{x_{t}} u\right\rangle-\left\langle\uparrow_{y_{t}}^{x_{t}}, \nabla_{y_{t}} u\right\rangle \leq-\frac{u\left(y_{t}\right)-u\left(x_{t}\right)}{\ell(t)}-\frac{u\left(x_{t}\right)-u\left(y_{t}\right)}{\ell(t)}=0 .
$$


Thus we get $\ell(t) \leq \ell(s)$. Since $\left\langle\uparrow_{x_{t}}^{y_{t}}, \alpha^{+}(t)\right\rangle+\left\langle\uparrow_{y_{t}}^{x_{t}}, \beta^{+}(t)\right\rangle=\left\langle\uparrow_{x_{t}}^{y_{t}}, \nabla_{x_{t}} u\right\rangle+\left\langle\uparrow_{y_{t}}^{x_{t}}, \nabla_{y_{t}} u\right\rangle \leq 0$, we have

$$
\ell^{-}(t) \leq-\left\langle\uparrow_{x_{t}}^{y_{t}}, \alpha^{-}(t)\right\rangle-\left\langle\uparrow_{y_{t}}^{x_{t}}, \beta^{-}(t)\right\rangle=\left\langle\uparrow_{x_{t}}^{y_{t}}, \alpha^{+}(t)\right\rangle+\left\langle\uparrow_{y_{t}}^{x_{t}}, \beta^{+}(t)\right\rangle \leq 0
$$

Thus $\ell(t) \geq \ell(s)$.

Now we prove (C). By Toponogov comparison and (5.2), we get that

$$
\begin{aligned}
d^{2}\left(x_{t}, y\right) & \leq d^{2}(x, y)+t^{2}-2 t \cdot d(x, y) \cdot\left\langle\uparrow_{x}^{y}, \nabla_{x} u\right\rangle \\
& \leq d^{2}(x, y)+t^{2}-2 t \cdot(u(y)-u(x)) .
\end{aligned}
$$

Start with $x, y \in u^{-1}(\xi)$. Fix $y$ and flow $x$ by time $t$. By (5.5), we get

$$
d^{2}\left(x_{t}, y\right) \leq d^{2}(x, y)+t^{2} .
$$

Fix $x_{t}$ and flow $y$ by time $t$. That is, in (5.5), substitute $y$ by $x_{t}, x$ by $y$ and $x_{t}$ by $y_{t}$. We get

$$
\begin{aligned}
d^{2}\left(y_{t}, x_{t}\right) & \leq d^{2}\left(y, x_{t}\right)+t^{2}-2 t \cdot\left(u\left(x_{t}\right)-u(y)\right) \\
& \leq d^{2}\left(x_{t}, y\right)+t^{2}-2 t \cdot\left(u\left(x_{t}\right)-u(x)\right) \\
& =d^{2}\left(x_{t}, y\right)-t^{2} .
\end{aligned}
$$

Combine (5.6) and (5.7). We have

$$
d^{2}\left(x_{t}, y_{t}\right) \leq d^{2}\left(x_{t}, y\right)-t^{2} \leq d^{2}(x, y) .
$$

By (B), we have $d\left(x_{t}, y_{t}\right)=d(x, y)$. Thus the Pythagorean Theorem $d^{2}\left(x_{t}, y\right)=d^{2}(x, y)+t^{2}$ follows.

Suppose that the statement has been proved for $k$. Apply the previous argument on the 0 -splitting function $u_{k+1}: B_{5}(p) \rightarrow \mathbb{R}$, we have that $B_{1}(p)$ is isometric to a ball in $Z \times \mathbb{R} \in$ Alex $^{n}(0)$, and it splits off $\mathbb{R}^{1}$ along the direction $\nabla u_{k+1}$. Note that $Z \times \mathbb{R} \in \operatorname{Alex}^{n}(0)$ if and only if $Z \in \operatorname{Alex}^{n-1}(0)$. Thus restricted on $Z \times\{0\} \in \operatorname{Alex}^{n-1}(0)$, the map $\left(u_{1}, \ldots, u_{k}\right)$ is $(k, 0)$-splitting. Then the result follows from the inductive hypothesis on $k$.

Assertion (ii) is a consequence of (iii). The proof of (iii) is standard, for instance if $u_{i}(x)=d\left(a_{i}, x\right)$, then by the arguments used in Sections $5.6-5.7$ in [2] we have that $u=\left(u_{1}, \ldots, u_{k}\right)$ is a $(k, 100 \delta)$-splitting function on $B_{\delta R}(p)$, if $\delta=\delta(n)$ is chosen sufficiently small.

The following statement is an easy consequence of Proposition 5.1.

Corollary 5.2. For any $n, k \in \mathbb{N}$ and $\epsilon>0$, there exists $\delta=\delta(n, \epsilon)>0$ so that if $X \in$ Alex $^{n}(-\delta)$ and $B_{5}(p)$ is $(k, \delta)$-splitting, then $B_{r}(x)$ is $(k, \epsilon)$-splitting for every $x \in B_{1}(p)$ and every $r \in(0,1]$.

5.2. Strong and weak singularity. In this Subsection we discuss the relations between the strong and weak quantitative singular sets. In fact, they are equivalent in some sense for Alexandrov spaces.

Define weak singular sets

$$
\widetilde{\mathcal{S}}_{\epsilon, r}^{k}(X)=\left\{x \in X: B_{s}(x) \text { is not }(k+1, \epsilon) \text {-splitting for every } s \in(r, 1]\right\} .
$$


It's clear that $\widetilde{\mathcal{S}}_{\epsilon, r}^{k}(X) \subseteq \mathcal{S}_{\epsilon, r}^{k}(X)$.

By Corollary 5.2, we have

Proposition 5.3. For any $n, \epsilon>0$, there exists $\delta(n, \epsilon)>0$ such that for any $X \in \operatorname{Alex}^{n}(-\delta)$ and $0<r \leq 1$, we have

$$
\widetilde{\mathcal{S}}_{\epsilon, r}^{k}(X) \subseteq \mathcal{S}_{\epsilon, r}^{k}(X) \subseteq \widetilde{\mathcal{S}}_{\delta, r}^{k}(X)
$$

The quantitative singular sets defined for the Ricci cases in [5] is as follows. Note that we do not use it in this paper and it may be skipped, we are presenting this for comparison sake to the Ricci curvature context.

Definition 5.4 (Quantitative symmetric).

(1) Given a metric space $Y$ and $k \in \mathbb{N}$, we say that $Y$ is $k$-symmetric if $Y \equiv \mathbb{R}^{k} \times C(\Sigma)$ for some metric space $\Sigma$.

(2) Given $x \in X$ we say that $B_{r}(x)$ is $(k, \epsilon)$-symmetric if there exists a $k$-symmetric space $Y$ such that $d_{G H}\left(B_{r}(x), B_{r}(y)\right) \leq \epsilon r$, where $y \in Y$ is a cone point.

Define

$$
\mathcal{W S}_{\epsilon, r}^{k}(X) \equiv\left\{x \in X: B_{s}(x) \text { is not }(k+1, \epsilon) \text {-symmetric, for every } s \in(r, 1]\right\} .
$$

It's clear that $\widetilde{\mathcal{S}}_{\epsilon, r}^{k}(X) \subseteq \mathcal{W} \mathcal{S}_{\epsilon, r}^{k}(X)$.

The following is an easy lemma, by a standard contradiction argument.

Lemma 5.5. For each $n \in \mathbb{N}$ and $\epsilon>0$ there exists $\delta(n, \epsilon)>0$ such that the following holds for any metric space $(X, p)$. If $B_{r}(p)$ is both $(0, \delta)$-symmetric and $(k, \delta)$-splitting, then $B_{r}(p)$ is $(k, \epsilon)$-symmetric.

Proposition 5.6. For any $\epsilon>0$, there exist $\eta(n, \epsilon)$ and $\delta(n, \epsilon)>0$ such that for any $X \in$ Alex $^{n}(-\delta)$ and $0<r \leq 1$, we have

$$
\mathcal{W} \mathcal{S}_{\epsilon, \eta r}^{k}(X) \subseteq \widetilde{\mathcal{S}}_{\delta, r}^{k}(X) \subseteq \mathcal{W} \mathcal{S}_{\delta, r}^{k}(X)
$$

Proof. If $x \notin \widetilde{\mathcal{S}}_{\delta, r}^{k}(X)$, then $B_{s}(x)$ is $(k+1, \delta)$-splitting for some $s \geq r$. By Corollary 5.2, we have that $B_{t}(x)$ is $\left(k+1, \delta_{1}\right)$-splitting for all $t \in\left(0, \frac{1}{5} r\right]$. On the other hand, by Lemma 4.6, there exists $\eta\left(n, \delta_{1}\right)>0$ and $r_{x} \in\left[\eta r, \frac{1}{5} r\right]$ such that $B_{r_{x}}(x)$ is $\left(0, \delta_{1}\right)$-symmetric. Due to Lemma 5.5, with appropriately selected $\delta$ and $\delta_{1}$, we have that $B_{r_{x}}(x)$ is $(k+1, \epsilon)$-symmetric. Therefore, $x \notin \mathcal{W} \mathcal{S}_{\epsilon, \eta r}^{k}(X)$.

Remark 5.1. Our notion of quantitative splitting for Alexandrov spaces is also equivalent to those defined using strainers. In particular, there exists $0<\delta_{1}(n, \epsilon)<\delta_{2}(n, \epsilon)$ so that

$$
\mathcal{S}_{\epsilon, r / 5}^{k}(X) \subseteq\left\{x \in X: x \text { does not admit any }\left(k+1, \delta_{2}\right) \text {-strainer with size } \geq r\right\} \subseteq \mathcal{S}_{\delta_{1}, r}^{k}(X)
$$

for any $X \in \operatorname{Alex}^{n}\left(-\delta_{1}\right)$ and $0<r \leq 1$. 
Remark 5.2. By a similar argument, one can show that if $X$ is a $v$-non-collapsed limit of $n$-dimensional manifolds with Ric $\geq-1$, then there exist $\eta_{i}(n, \epsilon, v)>0, i=1,2$, such that

$$
\mathcal{W} \mathcal{S}_{\epsilon, \eta_{1} r}^{k}(X) \subseteq \widetilde{\mathcal{S}}_{\eta_{2}, r}^{k}(X) \subseteq \mathcal{S}_{\eta_{2}, r}^{k}(X) \text {. }
$$

However, the statement in the form $\mathcal{S}_{\epsilon, \eta_{1} r}^{k}(X) \subseteq \mathcal{W S}_{\eta_{2}, r}^{k}(X)$ doesn't hold for the Ricci case.

5.3. Dimension reduction. Note in a metric cone $C(\Sigma)$ that the tangent cone at any point $p \in C(\Sigma)$ away from the cone point splits off an extra $\mathbb{R}$-factor in comparison to $C(\Sigma)$. This is the basis of Federer dimension reduction. The following lemma is a quantitative version of this on Alexandrov spaces.

Lemma 5.7. For any $n, k \in \mathbb{N}$ and $\epsilon>0$, there exists $\delta=\delta(n, \epsilon)$ and $\beta=\beta(n, \epsilon)>0$ such that the following holds for any $(X, p) \in$ Alex $^{n}(-\delta)$ and $(k, \delta)$-splitting function $u=\left(u_{1}, \ldots, u_{k}\right): B_{50}(p) \rightarrow \mathbb{R}^{k}$. Let $x \in B_{1}(p)$ and $y \in X$ with $d(x, y)=r>0$.

(i) If $T_{x}^{\delta}(r, 2 r)=0$ and $d(x, y)-d(u(x), u(y))>\epsilon r$, then $B_{s}(y)$ is $(k+1, \epsilon)$-splitting for every $0<s \leq \beta r$.

(ii) If $T_{x}^{\delta}(r, 2 r)=0$ and $B_{s}(y)$ is not $(k+1, \epsilon)$-splitting for some $0<s \leq \beta r$, then

$$
|d(u(x), u(y))-d(x, y)| \leq \epsilon d(x, y) .
$$

Proof. We only need to prove (i) since it is equivalent to (ii), taking in account that $|\nabla u|<1+\delta$. Let $\delta=\delta(n, \epsilon), \delta_{i}=\delta_{i}(n, \epsilon)$ be constants with $0<\delta<\delta_{1}<\delta_{2}<\cdots<\epsilon$.

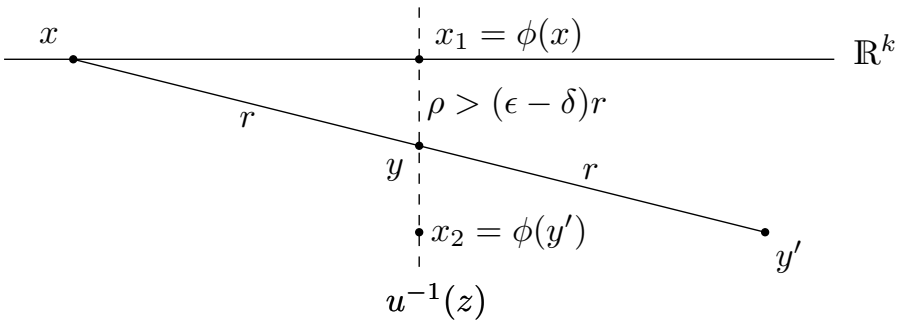

FIGURE 1.

Let us take $z \equiv u(y)$. Choosing $\delta(n, \epsilon)>0$ small we have by Proposition 5.1 that there exists $\phi: B_{10 r}(x) \rightarrow$ $u^{-1}(z)$ such that $(u, \phi): B_{10 r}(x) \rightarrow \mathbb{R}^{k} \times u^{-1}(z)$ is a $\delta_{1} r$-isometry. Let $x_{1}=\phi(x) \in u^{-1}(z)$ and $\rho=d\left(x_{1}, y\right)$.

We first find a splitting function along the slice $u^{-1}(z)$, using that $y$ is away from the cone point $x_{1}$. Because $T_{x}^{\delta}(r, 2 r)=0$, there exists $y^{\prime} \in X$ so that

$$
\left|d\left(y, y^{\prime}\right)-r\right| \leq \delta_{1} r, \quad\left|d\left(x, y^{\prime}\right)-2 r\right| \leq \delta_{1} r .
$$

Let $x_{2}=\phi\left(y^{\prime}\right) \in u^{-1}(z)$. Combine (5.14), $d(x, y)=r$ and the $\delta r$-splitting structure on $B_{10 r}(x)$. We have

$$
\left|d\left(x_{2}, y\right)-\rho\right| \leq 4 \delta_{1} r, \quad\left|d\left(x_{1}, x_{2}\right)-2 \rho\right| \leq 4 \delta_{1} r .
$$


To see the maximal scales that $B_{s}(y)$ splits, we need a lower bound of $\rho$. Because $(u, \phi)$ is a $\delta_{1} r$-isometry and by the assumptions, we have

$$
\begin{aligned}
\rho=d\left(x_{1}, y\right) & \geq d(x, y)-d\left(x, x_{1}\right) \\
& \geq d(x, y)-d\left((u, \phi)(x),(u, \phi)\left(x_{1}\right)\right)-\delta_{1} r \\
& =d(x, y)-d((u(x), \phi(x)),(u(y), \phi(x)))-\delta_{1} r \\
& =d(x, y)-d(u(x), u(y))-\delta_{1} r \\
& >\left(\epsilon-\delta_{1}\right) r .
\end{aligned}
$$

Choosing $\delta_{1}$ small and by (5.15), we have that $\left\{x_{1}, x_{2}\right\}$ forms a $\left(1, \delta_{2}\right)$-strainer. Thus $u_{k+1}(q) \equiv d\left(q, x_{1}\right)$ is a $\left(1, \delta_{3}\right)$-splitting map on $B_{\frac{1}{5} \epsilon r}(y)$.

By the $\delta_{1}$-almost splitting structure of $B_{10}(p)$ and the Toponogov comparison using (5.15), we have

$$
\left|\left\langle\nabla_{q} u_{i}, \nabla_{q} u_{k+1}\right\rangle\right|<\delta_{3} .
$$

for any $q \in B_{\frac{1}{5} \epsilon r}(y)$ and every $i=1,2, \ldots k$. Thus the function $\left.\left(u, u_{k+1}\right)\right|_{B_{\frac{1}{5} \epsilon r}}(y)$ is a $\left(k+1, \delta_{3}\right)$-splitting map. By Proposition 5.1 (i), $B_{s}(y)$ is $\epsilon$-splitting for every $0<s \leq \frac{1}{50} \epsilon r$.

Using Lemma 5.7, we can prove the rectifiability.

Proof of Theorem 1.6. Note that $\left(\mathcal{S}_{\epsilon}^{k} \backslash \mathcal{S}_{\delta}^{k-1}\right) \cap B_{1}$ can be covered by countably many balls $\left\{B_{\delta r_{i}}\left(x_{i}\right)\right\}$ with $x_{i} \notin \mathcal{S}_{\delta, 50 r_{i}}^{k}$. That is, $B_{50 r_{i}}\left(x_{i}\right)$ is $(k, \delta)$-splitting. By Proposition 5.1, for each of $B_{\delta r_{i}}\left(x_{i}\right)$, there exists a $\delta_{1}$ splitting map $u_{i}: B_{50 \delta r_{i}}\left(x_{i}\right) \rightarrow \mathbb{R}^{k}$. Note that $\operatorname{dim}_{\mathcal{H}}\left(\mathcal{S}_{\delta}^{k-1}\right) \leq k-1$. Thus it suffices to prove the following statement. There exists $\delta=\delta(n, \epsilon)>0$, such that if there is a $(k, \delta)$-splitting map $u: B_{50}(p) \rightarrow \mathbb{R}^{k}$, then $\mathcal{S}_{\epsilon}^{k} \cap B_{1}(p)$ is $k$-rectifiable.

Let $\delta(n, \epsilon)>0$ be determined later. Recall that by Lemma 2.2, for every point $x \in X$, the number of $\delta$-bad scales is at most $N(n, \delta)$. For each $x \in B_{1}(p)$, let $s_{x} \in(0,1]$ be the minimum of 1 and the smallest $\delta$-bad scale at $x$. Put $\Gamma_{x}^{t}=B_{t / 2}(x) \cap \mathcal{S}_{\epsilon}^{k} \cap\left\{y \in B_{1}(p): s_{y}>2 t\right\}$. We claim that for any $t>0$, the map $\left.u\right|_{\Gamma_{x}^{t}}: \Gamma_{x}^{t} \rightarrow \mathbb{R}^{k}$ is bi-Lipschitz onto its image. Once the claim is proved, we have that $\mathcal{S}_{\epsilon}^{k} \cap\left\{y \in B_{1}(p): s_{y}>2 t\right\}=\cup_{x \in B_{1}(p)} \Gamma_{x}^{t}$ is $k$-rectifiable. Therefore,

$$
\mathcal{S}_{\epsilon}^{k} \cap B_{1}(p)=\bigcup_{t>0}\left(\mathcal{S}_{\epsilon}^{k} \cap\left\{y \in B_{1}(p): s_{y}>2 t\right\}\right)
$$

is rectifiable.

Now we prove the claim. Let $x_{1}, y_{1} \in \Gamma_{x}^{s}$. Then $d\left(x_{1}, y_{1}\right) \leq t<s_{x_{1}} / 2$. Because $\operatorname{Bad}^{\delta}\left(x_{1}\right) \cap\left[0, s_{x_{1}}\right)=\varnothing$, by Proposition 4.1, we have $T_{x_{1}}^{\delta}(0,2 t)=T_{x_{1}}^{\delta}\left(0, s_{x_{1}}\right)=0$. Note that $y_{1} \in \mathcal{S}_{\epsilon}^{k}$ and thus $B_{\rho}\left(y_{1}\right)$ is not $(k+1, \epsilon)$ splitting for every $\rho \in(0,1]$. By Lemma 5.7 (ii), we have

$$
\left|d\left(u\left(x_{1}\right), u\left(y_{1}\right)\right)-d\left(x_{1}, y_{1}\right)\right| \leq \epsilon d\left(x_{1}, y_{1}\right) .
$$




\section{Packing Estimates}

We prove Theorem 1.3 in this section. The following is the key lemma.

Lemma 6.1. For any $n \in \mathbb{N}$ and $\epsilon>0$, there exist $\delta(n, \epsilon)>0$ and $\beta(n, \epsilon)>0$ so that the following holds for any $(X, p) \in$ Alex $^{n}(-\delta)$. Suppose $u: B_{50}(p) \rightarrow \mathbb{R}^{k}$ is a $(k, \delta)$-splitting function, and let $\left\{B_{r_{i}}\left(x_{i}\right)\right\}$ with $i \in \mathbb{I}$ be a disjoint collection of balls living on a fixed level set $x_{i} \in u^{-1}(z) \cap B_{1}(p)$ for some $z \in \mathbb{R}^{k}$. Then if $x_{i} \in \mathcal{S}_{\epsilon, \beta r_{i}}^{k}$ we have the estimate $|\mathbb{I}|<N(n, \epsilon)$.

Proof. We will construct a sequence Vitali coverings of $u^{-1}(z) \cap B_{1}(p)$, which "converges" to $\left\{B_{r_{i}}\left(x_{i}\right), i \in \mathbb{I}\right\}$. The constants $\delta(n, \epsilon), \eta(n, \epsilon)>0$ and $\lambda>0$ will be determined later.

(Step 1.) Let $\bar{B}_{\rho}$ be an arbitrary closed ball with $W \subseteq \bar{B}_{\rho}$ be a closed subset and $\mathbb{I}(W)=\left\{i \in \mathbb{I}: x_{i} \in W\right\}$. For $x \in W$ and $0<\epsilon, s \leq 1$, define function

$$
\sigma(x, \epsilon, s)= \begin{cases}\inf \left\{\tau: T_{x}^{\epsilon}(\tau s, 2 s)=0\right\}, & \text { if } \quad T_{x}^{\epsilon}(s, 2 s)=0 \\ 1, & \text { otherwise }\end{cases}
$$

By Lemma 4.6, for each $0<\lambda<4^{-1}$ there exists $\eta=\eta(n, \epsilon, \lambda)>0$ such that for any $x \in W$, there exists $r_{x} \in[\eta \rho, \rho]$ such that $T_{x}^{\epsilon}\left(\lambda r_{x}, 2 r_{x}\right)=0$. Therefore, we have

$$
\lambda_{x} \equiv \sigma\left(x, \epsilon, r_{x}\right) \leq \lambda
$$

Define $\mathcal{F}(W)=\left\{i \in \mathbb{I}(W): r_{i} \geq \frac{1}{10} \eta \rho\right\}$ and $\mathcal{F}^{c}(W)=\mathbb{I}(W) \backslash \mathcal{F}(W)$. It is clear that $|\mathcal{F}(W)| \leq N(n, \eta)$, since $r_{i} \geq \frac{1}{10} \eta \rho$ and $W \subseteq \bar{B}_{\rho}$. Now because $r_{i}<\frac{1}{10} \eta \rho \leq \frac{1}{10} r_{x_{i}}$ for every $i \in \mathcal{F}^{c}(W)$, we have that $\left\{B_{\frac{1}{10}} r_{x_{i}}\left(x_{i}\right), i \in\right.$ $\left.\mathcal{F}^{c}(W)\right\}$ is a covering of $\cup_{i \in \mathcal{F}^{c}(W)} B_{r_{i}}\left(x_{i}\right)$. Let $\mathcal{G}(W) \subseteq \mathcal{F}^{c}(W)$ be a collection of indices so that $\left\{B_{\frac{1}{10} r_{x_{j}}}\left(x_{j}\right), j \in\right.$ $\mathcal{G}(W)\}$ covers $\cup_{i \in \mathcal{F}^{c}(W)} B_{r_{i}}\left(x_{i}\right)$, while $\left\{B_{\frac{1}{50}} r_{x_{j}}\left(x_{j}\right), j \in \mathcal{G}(W)\right\}$ are disjoint. It's clear that $|\mathcal{G}(W)| \leq N(n, \eta)$, since $r_{x_{i}} \geq \eta \rho$. Now we have

$$
\mathbb{I}(W) \subseteq \mathcal{F}(W) \cup\left(\bigcup_{j \in \mathcal{G}(W)} \mathbb{I}\left(B_{\frac{1}{10} r_{x_{j}}}\left(x_{j}\right)\right)\right),
$$

where $|\mathcal{F}(W)|+|\mathcal{G}(W)| \leq N(n, \eta)$.

Note that function $\sigma(x, \epsilon, s)$ is semi-continuous in $x$. That is, $\liminf _{z \rightarrow y} \sigma\left(z, \epsilon, r_{x}\right) \geq \sigma\left(y, \epsilon, r_{x}\right)$. For each $j \in \mathcal{G}(W)$, there exists $y_{j} \in \bar{B}_{\lambda_{x_{j}} r_{x_{j}}}\left(x_{j}\right) \cap W$ so that

$$
\sigma_{y_{j}} \equiv \sigma\left(y_{j}, \epsilon, r_{x_{j}}\right)=\inf \left\{\sigma\left(x, \epsilon, r_{x_{j}}\right): x \in \bar{B}_{\lambda_{x_{j}} r_{x_{j}}}\left(x_{j}\right)\right\} \leq \lambda_{x_{j}} \leq \lambda .
$$

We claim that $\mathcal{F}(W)$ and $\mathcal{G}(W)$ satisfy the following properties.

(1) $|\mathcal{F}(W)|+|\mathcal{G}(W)| \leq N(n, \eta)$.

(2) $\mathbb{I}(W)=\mathcal{F}(W) \cup\left(\bigcup_{j \in \mathcal{G}(W)} \mathbb{I}\left(B_{\lambda_{x_{j}} r_{x_{j}}}\left(x_{j}\right) \cap B_{\sigma_{y_{j}} r_{x_{j}}}\left(y_{j}\right)\right)\right)$.

(3) If $\sigma_{y_{j}}>0$, then for every $z \in \bar{B}_{\lambda_{x_{j}} r_{x_{j}}}\left(x_{j}\right) \cap \bar{B}_{\sigma_{y_{j}} r_{x_{j}}}\left(y_{j}\right)$

$$
\left|\operatorname{Bad}^{\epsilon}(z) \cap\left[\sigma_{y_{j}} r_{x_{j}}, 1\right]\right| \geq\left|\operatorname{Bad}^{\epsilon}(z) \cap[\rho, 1]\right|+1 .
$$


Statement (1) has been proved in the construction. To prove (2), we start with an obvious inclusion formula:

$$
\begin{gathered}
B_{\frac{1}{10} r_{x_{j}}}\left(x_{j}\right) \subseteq\left(A_{\lambda_{x_{j}} r_{x_{j}}}^{\frac{1}{10} r_{x_{j}}}\left(x_{j}\right) \cup A_{\sigma_{y_{j}} r_{x_{j}}}^{\frac{1}{10} r_{x_{j}}}\left(y_{j}\right)\right) \cup\left(\bar{B}_{\lambda_{x_{j}} r_{x_{j}}}\left(x_{j}\right) \cap \bar{B}_{\sigma_{y_{j}} r_{x_{j}}}\left(y_{j}\right)\right) \\
\cup\left(\bar{B}_{\lambda_{x_{j}} r_{x_{j}}}\left(x_{j}\right) \backslash \bar{B}_{r_{x_{j}}}\left(y_{j}\right)\right) \cup\left(\bar{B}_{\sigma_{x_{j}} r_{x_{j}}}\left(y_{j}\right) \backslash \bar{B}_{r_{x_{j}}}\left(x_{j}\right)\right) .
\end{gathered}
$$

Let $\lambda<\frac{1}{10}$ be a constant. Note that $d\left(x_{j}, y_{j}\right) \leq \sigma_{y_{j}} r_{x_{j}} \leq \lambda_{x_{j}} r_{x_{j}} \leq r_{x_{j}} / 10$. Thus we have $\bar{B}_{\lambda_{x_{j}} r_{x_{j}}}\left(x_{j}\right) \subseteq \bar{B}_{r_{x_{j}}}\left(y_{j}\right)$ and $\bar{B}_{\sigma_{y_{j}} r_{x_{j}}}\left(y_{j}\right) \subseteq \bar{B}_{r_{x_{j}}}\left(x_{j}\right)$. In particular we then have the better inclusion

$$
B_{\frac{1}{10} r_{x_{j}}}\left(x_{j}\right) \subseteq\left(A_{\lambda_{x_{j}} r_{x_{j}}}^{\frac{1}{10} r_{x_{j}}}\left(x_{j}\right) \cup A_{\sigma_{y_{j}} r_{x_{j}}}^{\frac{1}{10} r_{x_{j}}}\left(y_{j}\right)\right) \cup\left(\bar{B}_{\lambda_{x_{j}} r_{x_{j}}}\left(x_{j}\right) \cap \bar{B}_{\sigma_{y_{j}} r_{x_{j}}}\left(y_{j}\right)\right)
$$

It remains to show that

$$
\mathbb{I}\left(A_{\lambda_{x_{j}} r_{x_{j}}}^{\frac{1}{10} r_{x_{j}}}\left(x_{j}\right)\right)=\mathbb{I}\left(A_{\sigma_{y_{j}} r_{x_{j}}}^{\frac{1}{10} r_{x_{j}}}\left(y_{j}\right)\right)=\varnothing
$$

Suppose $\mathbb{I}\left(A_{\lambda_{x_{j}} r_{x_{j}}}^{\frac{1}{10} r_{x_{j}}}\left(x_{j}\right)\right) \neq \varnothing$. That is, there exists $i \in \mathcal{F}^{c}(W)$, so that $\lambda_{x_{j}} r_{x_{j}} \leq d\left(x_{i}, x_{j}\right) \leq \frac{1}{10} r_{x_{j}}$. Then from the definition of $r_{x_{j}}$ we have

$$
T_{x_{j}}^{\epsilon}\left(d\left(x_{i}, x_{j}\right), 2 d\left(x_{i}, x_{j}\right)\right)=0 .
$$

Now let $\beta=\beta(n, \epsilon)>0$ be the constant determined in Lemma 5.7. Because $B_{\beta r_{i}}\left(x_{i}\right)$ is not $(k+1, \epsilon)$-splitting and $r_{i} \leq d\left(x_{i}, x_{j}\right)$, the restricted map $\left.u\right|_{\left\{x_{i}, x_{j}\right\}}$ is $(1 \pm 4 \epsilon)$-bi-Lipschitz. This contradicts to the assumption $u\left(x_{i}\right)=u\left(x_{j}\right)=z$. The proof for $\mathbb{I}\left(A_{\sigma_{y_{j}} x_{x_{j}}}^{\frac{1}{10} r_{x_{j}}}\left(y_{j}\right)\right)=\varnothing$ is similar.

To prove (3), let $z \in \bar{B}_{\lambda_{x_{j}} r_{x_{j}}}\left(x_{j}\right) \cap \bar{B}_{\sigma_{y_{j}} r_{x_{j}}}\left(y_{j}\right) \in \mathcal{D}\left(W_{r}\right)$. By the definition of $\sigma_{y_{j}}$, we have $\sigma\left(z, \epsilon, r_{x_{j}}\right) \geq$ $\sigma_{y_{j}}>0$. Thus $T_{z}^{\epsilon}\left(\frac{1}{2} \sigma_{y_{j}} r_{x_{j}}, r_{x_{j}}\right)=1$. By the definition of bad scales, this implies $\left|\operatorname{Bad}^{\epsilon}(z) \cap\left[\sigma_{y_{j}} r_{x_{j}}, r_{x_{j}}\right]\right| \geq 1$. Then (3) follows since $\left[\sigma_{y_{j}} r_{x_{j}}, r_{x_{j}}\right] \subseteq\left[\sigma_{y_{j}} r_{x_{j}}, \rho\right]$.

(Step 2.) In this step we construct a covering of I inductively. Let the decomposition functions $\mathcal{F}$ and $\mathcal{G}$ be defined in Step 1. Begin with $W=B_{1}(p)$. Let $\mathcal{C}_{1}=\mathcal{F}(W)$ and $\mathcal{D}_{1}=\mathcal{G}(W)$. Suppose $\mathcal{C}_{k}$ and $\mathcal{D}_{k}$ have been constructed and satisfy the following $\left(A_{k}\right)-\left(C_{k}\right)$ :

$$
\begin{aligned}
& \left(A_{k}\right)\left|\mathcal{C}_{k}\right| \leq k N(n, \eta)^{k},\left|\mathcal{D}_{k}\right| \leq N(n, \eta)^{k} . \\
& \left(B_{k}\right) \mathbb{I}=\mathcal{C}_{k} \cup\left(\underset{j \in \mathcal{D}_{k}}{\cup} \mathbb{I}\left(B_{\lambda_{x_{j}} r_{x_{j}}}\left(x_{j}\right) \cap B_{\sigma_{y_{j}} r_{x_{j}}}\left(y_{j}\right)\right)\right) . \\
& \left(C_{k}\right)\left|\operatorname{Bad}^{\epsilon}(z) \cap\left[\sigma_{y_{j}} r_{x_{j}}, 1\right]\right| \geq k \text { for any } j \in \mathcal{D}_{k} \text { and } z \in B_{\lambda_{x_{j}} r_{x_{j}}}\left(x_{j}\right) \cap B_{\sigma_{y_{j}} r_{x_{j}}}\left(y_{j}\right) \text {, provided } \sigma_{y_{j}}>0 .
\end{aligned}
$$

For each $j \in \mathcal{D}_{k}$ and $W_{j}=\bar{B}_{\lambda_{x_{j}} r_{j}}\left(x_{j}\right) \cap \bar{B}_{\sigma_{y_{j}} r_{x_{j}}}\left(y_{j}\right)$, using the construction of Step 1 let

$$
\mathcal{C}_{k+1}=\mathcal{C}_{k} \cup\left(\cup_{j \in \mathcal{D}_{k}} \mathcal{F}\left(W_{j}\right)\right)
$$

and

$$
\mathcal{D}_{k+1}=\cup_{j \in \mathcal{D}_{k}} \mathcal{G}\left(W_{j}\right) .
$$


Now we prove $\left(A_{k+1}\right)-\left(C_{k+1}\right)$ for $\mathcal{C}_{k+1}$ and $\mathcal{D}_{k+1}$. By (1) in Step 1, we have $\left|\mathcal{F}\left(W_{j}\right)\right|+\left|\mathcal{G}\left(W_{j}\right)\right| \leq N(n, \eta)$. Thus

$$
\left|\mathcal{C}_{k+1}\right| \leq\left|\mathfrak{C}_{k}\right|+N\left|\mathcal{D}_{k}\right| \leq k N^{k}+N^{k+1} \leq(k+1) N^{k+1}
$$

and

$$
\left|\mathcal{D}_{k+1}\right| \leq N\left|\mathcal{D}_{k}\right| \leq N^{k+1} .
$$

Statements $\left(B_{k+1}\right)$ and $\left(C_{k+1}\right)$ follow from (2) and (3) respectively.

(Step 3.) By Lemma 2.2, the number of $\epsilon$-bad scales is at most $K=K(n, \epsilon)$. Thus due to $\left(C_{k}\right)$, we have $\mathcal{D}_{k}=\varnothing$ if $k>K$. Therefore, $\mathbb{I}=\mathcal{C}_{K}$ and $|\mathbb{I}|=\left|\mathcal{C}_{K}\right| \leq K N^{K}$.

Furthermore, we have the following theorem.

Theorem 6.2. For any $n \in \mathbb{N}, \epsilon>0$ and $\Lambda \geq 1$, there exist $\delta(n, \epsilon)>0$ and $\beta(n, \epsilon)>0$ so that the following holds for any $(X, p) \in$ Alex $^{n}(-1)$. Suppose that there is a $(k, \delta)$-splitting function $u: B_{50}(p) \rightarrow \mathbb{R}^{k}$. If $\left\{B_{r_{i}}\left(x_{i}\right)\right\}$ are disjoint and $B_{\beta \Lambda r_{i}}\left(x_{i}\right) \cap \mathcal{S}_{\epsilon, \beta \Lambda r_{i}}^{k} \neq \varnothing$ for all $i \in \mathbb{I}$, then for any $z \in \mathbb{R}^{k}$, we have

$$
\left|\left\{i \in \mathbb{I}: B_{\beta \Lambda r_{i}}\left(x_{i}\right) \cap u^{-1}(z) \neq \varnothing\right\}\right|<N(n, \epsilon, \Lambda) .
$$

Additionally, if $r_{i}=r$ with $B_{\Lambda r}\left(x_{i}\right) \cap \mathcal{S}_{\epsilon, \Lambda r}^{k} \neq \varnothing$ and $\left\{B_{r}\left(x_{i}\right)\right\}$ are disjoint for all $i \in \mathbb{I}$, then for any $z \in \mathbb{R}^{k}$, we have

$$
\left|\left\{i \in \mathbb{I}: B_{\Lambda r}\left(x_{i}\right) \cap u^{-1}(z) \neq \varnothing\right\}\right|<N(n, \epsilon, \Lambda) .
$$

Proof. Let $\bar{x}_{i} \in B_{\beta \Lambda r_{i}}\left(x_{i}\right) \cap u^{-1}(z)$ and $y_{i} \in B_{\beta \Lambda r_{i}}\left(x_{i}\right) \cap \mathcal{S}_{\epsilon, \beta \Lambda r_{i}}^{k}$. There exists $\eta(n, \epsilon)>0$ such that $\bar{x}_{i} \in \mathcal{S}_{\eta, 10 \beta \Lambda r_{i}}^{k}$, since $B_{10 \beta \Lambda r_{i}}\left(\bar{x}_{i}\right) \supseteq B_{\beta \Lambda r_{i}}\left(y_{i}\right)$ and $B_{\beta \Lambda r_{i}}\left(y_{i}\right)$ is not $(k, \epsilon)$-splitting. Moreover, we have that $B_{r_{i} / 2}\left(\bar{x}_{i}\right)$ are disjoint, because $B_{r_{i} / 2}\left(\bar{x}_{i}\right) \subseteq B_{r_{i}}\left(x_{i}\right)$. Estimate (6.10) follows by applying Lemma 6.1 to the collection $\left\{B_{r_{i} / 2}\left(\bar{x}_{i}\right)\right\}$.

To prove (6.11), one can go through the proof of Lemma 6.1 and (6.10) with small modifications, or use the following re-covering arguments. Let $r^{\prime}=r / \beta$. Then we have $B_{\beta \Lambda r^{\prime}}\left(x_{i}\right)=B_{\Lambda r}\left(x_{i}\right)$. The given conditions $B_{\Lambda r}\left(x_{i}\right) \cap \mathcal{S}_{\epsilon, \Lambda r}^{k} \neq \varnothing$ and $B_{\Lambda r}\left(x_{i}\right) \cap u^{-1}(z) \neq \varnothing$ are equivalent to $B_{\beta \Lambda r^{\prime}}\left(x_{i}\right) \cap \mathcal{S}_{\epsilon, \beta \Lambda r^{\prime}}^{k} \neq \varnothing$ and $B_{\beta \Lambda r^{\prime}}\left(x_{i}\right) \cap u^{-1}(z) \neq \varnothing$, respectively. The collection $\left\{B_{r^{\prime}}\left(x_{i}\right)\right\}$ is not disjoint, so we can't use (6.10) directly. However, note that if $B_{r^{\prime}}\left(x_{i}\right) \cap B_{r^{\prime}}\left(x_{j}\right) \neq \varnothing$, then $B_{r}\left(x_{j}\right) \subseteq B_{2 r^{\prime}}\left(x_{i}\right)$. Because $\left\{B_{r}\left(x_{i}\right)\right\}$ are disjoint, for every $i$, there are at most $N\left(n, r^{\prime} / r\right)=N(n, \beta)$ balls $B_{r^{\prime}}\left(x_{j}\right)$ such that $B_{r^{\prime}}\left(x_{i}\right) \cap B_{r^{\prime}}\left(x_{j}\right) \neq \varnothing$. Therefore, the collection $\left\{B_{r^{\prime}}\left(x_{i}\right)\right\}$ can be written as the union of $N(n, \beta)$ disjoint collections. Then the result follows from (6.10).

Let us now remark on a standard covering argument. Let $\mathfrak{B}$ be a collection of sets. The intersection number $\mathcal{N}(\mathcal{B})$ of $\mathfrak{B}$ is the minimum number $k$ so that $B_{1} \cap B_{2} \cap \cdots \cap B_{k+1}=\varnothing$ for any $B_{1}, B_{2}, \ldots, B_{k+1} \in \mathfrak{B}$. In particular, if $\mathcal{N}(\mathcal{B})=1$, then $\mathfrak{B}$ is a disjoint collection. We have the following easy lemma:

Lemma 6.3. Let $B_{R}(0) \subset \mathbb{R}^{k}$ and $\mathfrak{B}=\left\{B_{r_{i}}\left(x_{i}\right) \subseteq B_{R}(0)\right\}$ be a collection of balls. If the intersection number $\mathcal{N}(\mathfrak{B}) \leq N<\infty$, then $\sum r_{i}^{k}<N \cdot C(k) R^{k}$.

Now let us prove a local version of Theorem 1.3. 
Lemma 6.4 (Local packing estimate). For any $n \in \mathbb{N}, \epsilon>0, R \leq 1$ and $\Lambda \geq 1$, there exists $\delta(n, \epsilon)>0$ and $\beta(n, \epsilon)>0$ so that the following hold for any $(X, p) \in \operatorname{Alex}^{n}(-1)$, provided that $B_{500 R}(p)$ is $(k, \delta)$-splitting.

(i) If $x_{i} \in \mathcal{S}_{\epsilon, \beta r_{i}}^{k} \cap B_{R}(p)$ with $r_{i} \leq R$ and $\left\{B_{r_{i}}\left(x_{i}\right)\right\}$ are disjoint for all $i \in \mathbb{I}$, then $\sum_{i \in \mathbb{I}} r_{i}^{k}<C(n, \epsilon) R^{k}$.

(ii) If $x_{i} \in \mathcal{S}_{\epsilon, \Lambda r}^{k} \cap B_{R}(p)$ with $r \leq R$ and $\left\{B_{r}\left(x_{i}\right)\right\}$ are disjoint for all $i \in \mathbb{I}$, then $|\mathbb{I}|<C(n, \epsilon, \Lambda)(R / r)^{k}$.

Proof. We prove (i) only and the proof of (ii) is similar, modulo (6.11). By Proposition 5.1, there is a $\delta_{1}$-splitting map $u: B_{50 R}(p) \rightarrow \mathbb{R}^{k}$. Assume $u(p)=0^{k} \in \mathbb{R}^{k}$.

Consider the collection of balls $\mathfrak{B}=\left\{B_{\frac{1}{2} \beta r_{i}}\left(u\left(x_{i}\right)\right), i \in \mathbb{I}\right\}$ in $\mathbb{R}^{k}$. Because $u$ is 1-Lipschitz, we have that $B_{\frac{1}{2} \beta r_{i}}\left(u\left(x_{i}\right)\right) \subseteq B_{2 R}\left(0^{k}\right)$. Given $z \in \mathbb{R}^{k}$, let $\mathbb{I}_{z}=\left\{i \in \mathbb{I}: z \in B_{\frac{1}{2} \beta r_{i}}\left(u\left(x_{i}\right)\right)\right\}$. By Proposition 5.1 again, we have $u^{-1}(z) \cap B_{\beta r_{i}}\left(x_{i}\right) \neq \varnothing$. It follows from (6.10) that $\left|\mathbb{I}_{z}\right| \leq N(n, \epsilon)$. This shows that the intersection number $\mathcal{N}(\mathfrak{B}) \leq N(n, \epsilon)$. Then the desired result follows from Lemma 6.3 .

Now we prove Theorem 1.3 by showing the following stronger statement.

Theorem 6.5 (Packing estimate). Lemma 6.4 still holds if the splitting assumption is dropped.

Proof. We prove by induction on $k$. Let $0<\delta^{\prime}(n, \epsilon)<\delta(n, \epsilon)<\delta_{1}(n, \epsilon)<\epsilon$ be determined latter. The constant $C$ may vary line by line. Lemma 6.4 proves the case for $k=0$ as well as the case that $B_{500 R}(p)$ is $\left(k+1, \delta_{1}\right)$-splitting. Assume that (i) and (ii) are true for $k<n$. We will prove them for $k+1$, assuming that $B_{500 R}(p)$ is not $\left(k+1, \delta_{1}\right)$-splitting.

Not losing generality, assume $R=\frac{1}{500}$. That is, $B_{1}(p)$ is not $\left(k+1, \delta_{1}\right)$-splitting. We begin with a decomposition of $B_{1}(p)$. Let $R_{\alpha}=2^{-\alpha}, \alpha \in \mathbb{Z}$. Recall the definition of the weak $(k, \delta)$-singular set $\widetilde{\mathcal{S}}_{\delta, r}^{k}$ in (5.8). By Proposition 5.3, we have $B_{1}(p) \subseteq \widetilde{\mathcal{S}}_{\delta, 10}^{k}$. Thus

$$
B_{1}(p) \backslash \widetilde{\mathcal{S}}_{\delta}^{k} \subseteq \widetilde{\mathcal{S}}_{\delta, 10}^{k} \backslash \widetilde{\mathcal{S}}_{\delta}^{k} \subseteq \bigcup_{\alpha=-4}^{\infty}\left(\widetilde{\mathcal{S}}_{\delta, R_{\alpha}}^{k} \backslash \widetilde{\mathcal{S}}_{\delta, R_{\alpha+1}}^{k}\right)
$$

For each $\alpha$, let

$$
\left\{B_{\rho_{\alpha}}\left(y_{j}^{\alpha}\right), j \in \mathbb{J}_{\alpha}\right\} \subseteq\left\{B_{\frac{1}{20} R_{\alpha}}(y), y \in \widetilde{\mathcal{S}}_{\delta, R_{\alpha}}^{k} \backslash \widetilde{\mathcal{S}}_{\delta, R_{\alpha+1}}^{k}\right\}
$$

be a Vitali covering of $\left(\widetilde{\mathcal{S}}_{\delta, R_{\alpha}}^{k} \backslash \widetilde{\mathcal{S}}_{\delta, R_{\alpha+1}}^{k}\right)$, for which $\left\{B_{\frac{1}{5} \rho_{\alpha}}\left(y_{j}\right)\right\}$ are disjoint but $\left\{B_{\rho_{\alpha}}\left(y_{j}\right)\right\}$ is a covering. A useful property for this decomposition is that for each $y \in\left\{y_{j}^{\alpha}\right\}$, we have that $B_{20 \rho_{\alpha}}(y)=B_{R_{\alpha}}(y)$ is not $(k+1, \delta)$-splitting, but $B_{10 \rho_{\alpha}}(y)=B_{\frac{1}{2} R_{\alpha}}(y)$ is $(k+1, \delta)$-splitting.

We first prove (ii), which will be needed in the proof of (i). By the inductive hypothesis, we only need to consider the collection of balls $\left\{B_{r}\left(x_{i}\right): x_{i} \in \mathcal{S}_{\epsilon, \Lambda r}^{k+1}\right.$ but $\left.x_{i} \notin \mathcal{S}_{\delta^{\prime}, r}^{k}\right\}$, where $\delta^{\prime}=\delta^{\prime}(n, \delta)>0$ will be determined latter. For each $j \in \mathrm{J}_{\alpha}$, because $y_{j}^{\alpha} \in \widetilde{\mathcal{S}}_{\delta, R_{\alpha}}^{k} \subseteq \mathcal{S}_{\delta, R_{\alpha}}^{k}$, by the inductive hypothesis, we have an upper bound on the number of these balls:

$$
\left|\mathbb{J}_{\alpha}\right| \leq C(n, \epsilon) R_{\alpha}^{-k}
$$


Recall that $x_{i} \in \mathcal{S}_{\epsilon, \Lambda r}^{k} \cap B_{1 / 500}(p)$ with $r \leq 1 / 500$ and $\left\{B_{r}\left(x_{i}\right)\right\}$ are disjoint. Given $j \in \mathbb{J}_{\alpha}$, let $\mathbb{I}_{j}^{\alpha}=\{i$ : $\left.x_{i} \in B_{\rho_{\alpha}}\left(y_{j}^{\alpha}\right)\right\}$. We claim that if $\rho_{\alpha}<r / 1000$, then $\mathbb{I}_{j}^{\alpha}=\varnothing$ for every $j$. Suppose $\rho_{\alpha}<r / 1000$ but there is $i \in \mathbb{I}_{j}^{\alpha}$ for some $j$. Note then that $R_{\alpha}=20 \rho_{\alpha}<\frac{1}{50} r$, we have $B_{r / 5}\left(x_{i}\right) \supseteq B_{20 \rho_{\alpha}}\left(y_{j}^{\alpha}\right)$. Because $B_{20 \rho_{\alpha}}\left(y_{j}^{\alpha}\right)$ is not $(k+1, \delta)$-splitting, we have $x_{i} \in \mathcal{S}_{\delta^{\prime}, r}^{k}$, for some $\delta^{\prime}(n, \delta)>0$. This contradicts to the assumptions.

Now for each $i \in \mathbb{I}_{j}^{\alpha}$, we have $x_{i} \in \mathcal{S}_{\epsilon, \Lambda r}^{k+1}, r \leq 1000 \rho_{\alpha}=50 R_{\alpha}$, and $B_{10 \rho_{\alpha}}\left(y_{j}^{\alpha}\right)=B_{\frac{1}{2} R_{\alpha}}(y)$ is $(k+1, \delta)$ splitting. By Lemma 6.4 (ii) we have

$$
\left|\mathbb{I}_{j}^{\alpha}\right| \leq C(n, \epsilon, \Lambda)\left(R_{\alpha} / r\right)^{k+1}
$$

Because $\cup_{\alpha \geq-4}\left\{B_{\rho_{\alpha}}\left(y_{j}^{\alpha}\right)\right\}$ is a covering of $B_{1}(p) \backslash \widetilde{\mathcal{S}}_{\delta}^{k} \supseteq B_{1}(p) \backslash \widetilde{\mathcal{S}}_{\delta^{\prime}}^{k} \supseteq\left\{x_{i}: i \in \mathbb{I}\right\}$, by (6.14) and (6.15), we have

$$
\begin{aligned}
|\mathbb{I}| & \leq \sum_{\frac{1}{50} r \leq R_{\alpha} \leq 10} \sum_{j \in \mathbb{J}_{\alpha}}\left|\mathbb{I}_{j}^{\alpha}\right| \\
& \leq \sum_{\alpha=-4}^{\infty} C(n, \epsilon, \Lambda) R_{\alpha}^{-k}\left(R_{\alpha} / r\right)^{k+1} \leq C(n, \epsilon, \Lambda) r^{-(k+1)}
\end{aligned}
$$

We prove (i) in a similar way. By the inductive hypothesis, we only need to consider the balls $\left\{B_{r_{i}}\left(x_{i}\right)\right.$ : $x_{i} \in \mathcal{S}_{\epsilon, \beta r_{i}}^{k+1}$ but $\left.x_{i} \notin \mathcal{S}_{\delta^{\prime}, \beta r_{i}}^{k}\right\}$, for some $\delta^{\prime}(n, \delta)>0$. Given $j \in \mathbb{J}_{\alpha}$, let $\mathbb{I}_{j}^{\alpha}=\left\{i: x_{i} \in B_{\rho_{\alpha}}\left(y_{j}^{\alpha}\right)\right\}$. We claim that for every $i \in \mathbb{I}_{j}^{\alpha}$, we have $r_{i} \leq \frac{1000}{\beta} \rho_{\alpha}$. If this is not true, then $B_{\beta r_{i} / 5}\left(x_{i}\right) \supseteq B_{20 \rho_{\alpha}}\left(y_{j}^{\alpha}\right)$. Because $B_{20 \rho_{\alpha}}\left(y_{j}^{\alpha}\right)$ is not $(k+1, \delta)$-splitting, we have that $B_{\beta r_{i}}\left(x_{i}\right)$ is not $\left(k+1, \delta^{\prime}\right)$-splitting for some $\delta^{\prime}=\delta^{\prime}(n, \delta)>0$. Thus $x_{i} \in \mathcal{S}_{\delta^{\prime}, \beta r_{i}}^{k}$, which contradicts to the assumptions.

Note that $x_{i} \in \mathcal{S}_{\epsilon, \beta r_{i}}^{k+1}$ and $B_{10 \rho_{\alpha}}\left(y_{j}^{\alpha}\right)=B_{\frac{1}{2} R_{\alpha}}(y)$ is $(k+1, \delta)$-splitting. We can apply Lemma 6.4 (i) and get

$$
\sum_{i \in \mathbb{I}_{j}^{\alpha}} r_{i}^{k+1} \leq C(n, \epsilon) \rho_{\alpha}^{k+1}=C(n, \epsilon) R_{\alpha}^{k+1} .
$$

Note that (6.14), which was proved in the course of proving (ii), still holds. Combine (6.14) and (6.16). We have

$$
\begin{aligned}
\sum_{i \in \mathbb{I}} r_{i}^{k+1} & \leq \sum_{\alpha=-4}^{\infty} \sum_{j \in \mathbb{J}_{\alpha}} \sum_{i \in \mathbb{I}_{j}^{\alpha}} r_{i}^{k+1} \\
& \leq \sum_{\alpha=-4}^{\infty} \sum_{j \in \mathbb{J}_{\alpha}} C(n, \epsilon) R_{\alpha}^{k+1} \\
& \leq \sum_{\alpha=-4}^{\infty} C(n, \epsilon) R_{\alpha}^{-k} R_{\alpha}^{k+1} \\
& \leq \sum_{\alpha=-4}^{\infty} C(n, \epsilon) R_{\alpha} \leq C(n, \epsilon) .
\end{aligned}
$$




\section{ShaRpNess OF THE RECTIFIABILITY}

In this section we prove Theorem 1.7. Let us begin with a smoothing lemma.

Lemma 7.1. Let $\mathbb{U} \subset \mathbb{R}^{n}$ be a compact convex subset and $f: \mathbb{U} \rightarrow \mathbb{R}$ be a strictly convex function. Let $\Omega=\cup_{i=1}^{\infty} \Omega_{i}$, where $\Omega_{i}$ are disjoint open convex subsets in $\mathbb{U}$. For any $\delta>0$, there exists a strictly convex function $F: \mathbb{U} \rightarrow \mathbb{R}$ such that the following hold.

(i) $\left.F\right|_{\Omega}$ is $C^{\infty}$.

(ii) $\left.F\right|_{U \backslash \Omega}=\left.f\right|_{U \backslash \Omega}$ and $|F-f|<\delta$ on $\Omega$,

(iii) For any $x \notin \Omega$ and any vector $v$, it holds that

$$
\lim _{t \rightarrow 0^{+}} \frac{F(x+t v)-F(x)}{t}=\lim _{t \rightarrow 0^{+}} \frac{f(x+t v)-f(x)}{t} .
$$

In particular, if $D f(x)$ exists at $x \notin \Omega$, then $D F(x)=D f(x)$.

Proof. Let

$$
\epsilon(x)=e^{-\frac{\delta}{d(x, U(\Omega)}}
$$

be an error function defined on $\Omega$. By Theorem 1.1 in [7], for each $i$, there exists a strictly $C^{\infty}$ convex function $g_{i}: \Omega_{i} \rightarrow \mathbb{R}$ such that for any $x \in \Omega_{i}$, we have

$$
\left|f(x)-g_{i}(x)\right| \leq \epsilon(x) .
$$

Let $F: \mathbb{U} \rightarrow \mathbb{R}$ be the gluing of all of $g_{i}$ and $\left.f\right|_{\mathbb{U} \backslash \Omega}$. That is,

$$
F(x)=\left\{\begin{array}{lll}
g_{i}(x), & \text { if } & x \in \Omega_{i} ; \\
f(x), & \text { if } & x \notin \Omega .
\end{array}\right.
$$

It is obvious that (i) and (ii) are satisfied. The following estimates (7.5) and (7.6) imply (iii). If $x, y \notin \Omega$, it is obvious that

$$
|| F(x)-F(y)|-| f(x)-f(y)||=0 .
$$

For any $x \notin \Omega$ and $y \in \Omega$, we have $y \in \Omega_{i}$ for some $i$ and thus

$$
\begin{aligned}
|| F(x)-F(y)|-| f(x)-f(y)|| & =|| f(x)-g_{i}(y)|-| f(x)-f(y)|| \\
& \leq\left|g_{i}(y)-f(y)\right| \\
& \leq e^{-\frac{\delta}{d(y, U(\Omega)}} \\
& \leq e^{-\frac{\delta}{d(x, y)}}
\end{aligned}
$$

It's clear that $F$ is strictly convex on each of $\Omega_{i}$. It remains to show that $F$ is strictly convex, moving out from $\Omega_{i}$. We need the following two lemmas which we will outline the proof latter. They are well known to the experts. 
Lemma 7.2. A Lipschitz function $h:[a, b] \rightarrow \mathbb{R}$ is convex if and only iffor any non-negative smooth function $\phi:[a, b] \rightarrow \mathbb{R}$, it holds that

$$
\int_{a}^{b} h^{\prime}(t) \phi^{\prime}(t) d t \leq h_{-}^{\prime}(b) \phi(b)-h_{+}^{\prime}(a) \phi(a) .
$$

Here $h_{ \pm}^{\prime}$ denote the one-sided derivatives.

Lemma 7.3. Let $h:[a, c] \rightarrow \mathbb{R}$ be a Lipschitz function and $b \in[a, c]$. If $\left.h\right|_{[a, b]}$ and $\left.h\right|_{[b, c]}$ are both convex functions and $h_{-}^{\prime}(b) \leq h_{+}^{\prime}(b)$, then $h$ is a convex function over $[a, c]$.

Now we show that $F$ is a convex function. It is obvious that $F$ is locally convex for any $x \notin \partial \Omega_{i}$. For $x \in \partial \Omega_{i}$, we show that $F$ is convex along each line passing through $x$ in $\mathbb{U}$. Let $\gamma(s)=x+s v, s \in(-\epsilon, \epsilon)$ be a unit speed geodesic in $\mathbb{U}$ and $h(s)=F(x+s v)$. By (7.1) and the fact that $f$ is convex, we have

$$
\begin{aligned}
h_{-}^{\prime}(0) & =\lim _{t \rightarrow 0^{-}} \frac{F(x+t v)-F(x)}{t} \\
& =\lim _{t \rightarrow 0^{-}} \frac{f(x+t v)-f(x)}{t} \leq \lim _{t \rightarrow 0^{+}} \frac{f(x+t v)-f(x)}{t} \\
& =\lim _{t \rightarrow 0^{+}} \frac{F(x+t v)-F(x)}{t}=h_{+}^{\prime}(0) .
\end{aligned}
$$

Then the convexity of $F$ follows from Lemma 7.3. By (iii), $F$ is also strictly convex.

Proof of Lemma 7.2. The necessity is obvious. To prove the sufficiency it is sufficient to verify

$$
\frac{h\left(t_{2}\right)-h\left(t_{1}\right)}{t_{2}-t_{1}}-\frac{h\left(t_{3}\right)-h\left(t_{2}\right)}{t_{3}-t_{2}} \leq 0
$$

for every $a \leq t_{1}<t_{2}<t_{3} \leq b$. This can be proved by a direct computation with $\phi(t)$ chosen as a smooth approximation to

$$
\psi(t)=\left\{\begin{array}{lll}
0, & \text { if } & t \leq t_{1}, \\
\frac{t-t_{1}}{t_{2}-t_{1}}, & \text { if } & t_{1}<t \leq t_{2}, \\
\frac{t_{3}-t}{t_{3}-t_{2}}, & \text { if } & t_{2}<t<t_{3}, \\
0, & \text { if } & t \geq t_{3} .
\end{array}\right.
$$

Proof of Lemma 7.3. By Lemma 7.2, for any non-negative smooth function $\phi:[a, b] \rightarrow[0, \infty)$, we have

$$
\begin{aligned}
& \int_{a}^{b} h^{\prime}(t) \phi^{\prime}(t) d t \leq h_{-}^{\prime}(b) \phi(b)-h_{+}^{\prime}(a) \phi(a), \\
& \int_{b}^{c} h^{\prime}(t) \phi^{\prime}(t) d t \leq h_{-}^{\prime}(c) \phi(c)-h_{+}^{\prime}(b) \phi(b) .
\end{aligned}
$$

Sum up the two inequalities and apply Lemma 7.2 again, we get the desired result. 
Proof of Theorem 1.7. Let $Z=\bar{B}_{1}(O) \subset \mathbb{R}^{2}$ be a closed unit disk centered at $p$. Fix $0<\delta<1$ and define a strictly concave function on $Z$ :

$$
f_{0}(z)=\left\{\begin{array}{lll}
\sqrt{d(z, \partial Z)} & \text { if } & d(z, \partial Z) \leq \frac{1}{4}, \\
\delta \cdot \sqrt{d(z, \partial Z)}+(1-\delta) \cdot \frac{1}{2} & \text { if } & d(z, \partial Z)>\frac{1}{4} .
\end{array}\right.
$$

Let $Z_{t}=\left\{z \in Z: f_{0}(z) \geq t\right\}$ be the sub-level set. We denote the subgraph of $f: Z \rightarrow \mathbb{R}^{+}$by

$$
G_{Z, f}=\{(z, t) \in Z \times \mathbb{R}: 0 \leq t \leq f(z)\} .
$$

Because $f_{0}$ is strictly concave, we have $X_{0}=G_{Z, f_{0}} \in \operatorname{Alex}^{3}(0)$ with boundary. See Figure 2 below. For

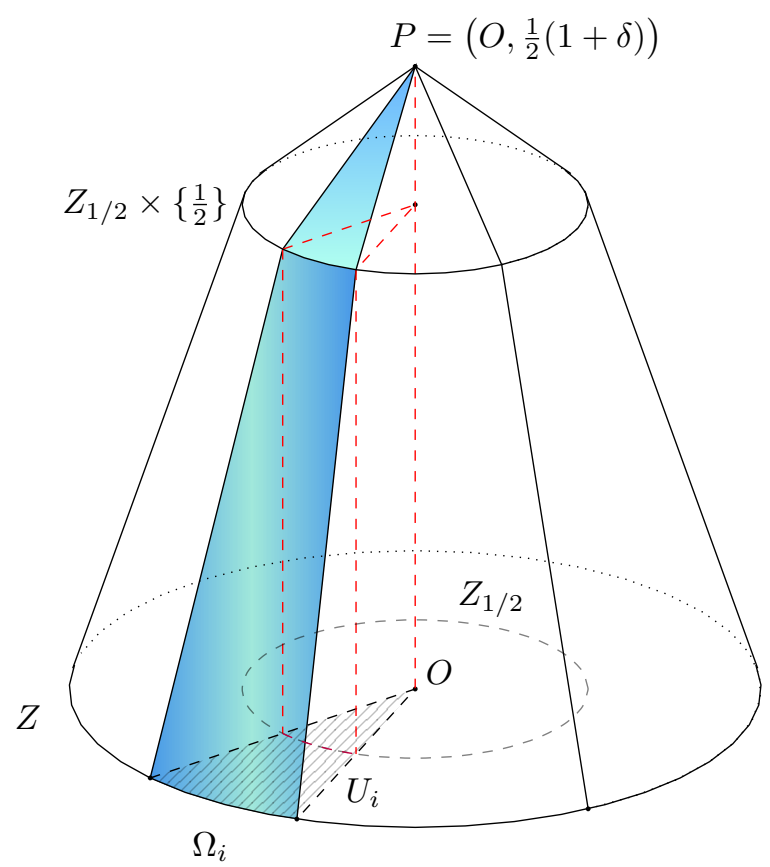

FiguRE 2. $X_{0}=G_{Z, f_{0}} \in \operatorname{Alex}^{3}(0)$

$\delta>0$ small the following hold:

(1) $\mathcal{S}\left(X_{0}\right)=\partial X_{0}$

(2) $\mathcal{S}_{\epsilon}^{1}\left(X_{0}\right) \backslash \mathcal{S}^{0}\left(X_{0}\right)=\left(\partial Z_{1 / 2} \times\left\{\frac{1}{2}\right\}\right) \cup(\partial Z \times\{0\})$

(3) $\mathcal{S}^{0}\left(X_{0}\right)=\{P\}=\left\{\left(O, \frac{1}{2}(1+\delta)\right)\right\}$ is the tip of the graph.

Not losing generality, let $T \subseteq \partial Z_{1 / 2}$ be any closed subset. Then $\partial Z_{1 / 2} \backslash T=\cup_{i=1}^{\infty} U_{i}$ is a union of disjoint open intervals. Let $\Omega_{i}$ be the open sectors in $Z$ corresponding to the arc $U_{i}$. That is, $\Omega_{i}=\{x \in$ $Z^{\circ}$ : ray $\left.\lambda \cdot \overrightarrow{O x} \cap U_{i} \neq \varnothing\right\}$, as the shaded region in Figure 2. Clearly, $\left\{\Omega_{i}\right\}$ is a collection of disjoint open convex sets. 
Now apply Lemma 7.1 to $f_{0}: Z \rightarrow \mathbb{R}$ on $\cup_{i=1}^{\infty} \Omega_{i}$ to obtain a strictly convex function $f_{1}: Z \rightarrow \mathbb{R}$ which is smooth on $\cup_{i=1}^{\infty} \Omega_{i}$ and $f_{1}=f_{0}$ away from $\cup_{i=1}^{\infty} \Omega_{i}$. Now consider the new subgraph $X_{1}=G_{Z, f_{1}} \in \operatorname{Alex}^{3}(0)$. Note that if $f_{1}$ is smooth at a point $x \in Z^{\circ}$, then the tangent cone of $X_{1}$ at $\left(x, f_{1}(x)\right) \in \partial X_{1}$ is a three dimensional half space. If $x \in \partial Z_{1 / 2} \backslash \cup_{i=1}^{\infty} \Omega_{i} \equiv T$, then the tangent cone at $\left(x, f_{1}(x)\right) \in \partial X_{1}$, which is isometric to the tangent cone of $X_{0}$ at $\left(x, f_{0}(x)\right)$, splits off only $\mathbb{R}^{1}$. Therefore we have

(4) $\mathcal{S}\left(X_{1}\right)=\partial X_{1}$,

(5) $\mathcal{S}_{\epsilon}^{1}\left(X_{1}\right) \backslash \mathcal{S}^{0}\left(X_{1}\right)=\left(T \times\left\{\frac{1}{2}\right\}\right) \cup(\partial Z \times\{0\})$,

(6) $\mathcal{S}^{0}\left(X_{1}\right)=\{P\}$ is the tip of the graph.

A similar, but less involved, smoothing procedure can be performed in a small neighborhood of $\partial Z \times\{0\}$ and $P$ so that the resulting space $X_{2} \in \operatorname{Alex}^{3}(0)$ satisfies

(7) $\mathcal{S}\left(X_{2}\right)=\partial X_{2}$,

(8) $\mathcal{S}_{\epsilon}^{1}\left(X_{2}\right)=T \times\left\{\frac{1}{2}\right\}$

(9) $\mathcal{S}^{0}\left(X_{2}\right)=\varnothing$.

Finally, we double $X_{2}$ and arrive at a boundary free space $Y \in \operatorname{Alex}^{3}(0)$ which satisfies

(10) $\mathcal{S}(Y)=\mathcal{S}_{\epsilon}^{1}(Y)=T \times\left\{\frac{1}{2}\right\}$;

(11) $\mathcal{S}^{0}(Y)=\varnothing$.

By performing similar smoothing procedures it is easy to verify that $Y$ can be realized as a non-collapsed limit of 3-dimensional manifolds with sec $\geq 0$.

\section{REFERENCES}

[1] S. Alexander, V. Kapovitch, A. Petrunin, Alexandrov geometry, a draft avaliable at www.math.psu.edu/petrunin.

[2] Y. Burago, M. Gromov, G. Perel'man, A.D. Alexandrov spaces with curvature bounded below, Uspekhi Mat. Nauk, 47:2 (1992), 3-51; translation in Russian Math. Surveys, 47:2 (1992), 1-58.

[3] J. Cheeger, T. Colding, On the structure of spaces with Ricci curvature bounded below I, J. Differential Geom., 45 (1997), 406-480.

[4] J. Cheeger, W. Jiang, A. Naber, Rectifiability of singular sets in noncollapsed spaces with Ricci curvature bounded below, arXiv: 1805.07988.

[5] J. Cheeger, A. Naber, Lower bounds on Ricci curvature and quantitative behavior of singular sets, Invent. Math., 191 (2013) no. 2, $321-339$.

[6] J. Cheeger, A. Naber, Regularity of Einstein manifolds and the codimension 4 conjecture, Ann. of Math. (2) 182 (2015), no. 3 , $1093-1165$.

[7] R. Greene, H. Wu, $C^{\infty}$ approximations of convex, subharmonic, and plurisubharmonic functions, Ann. Sci. Éc. Norm. Supér. (12) 1 (1979), $47-84$.

[8] V. Kapovitch, A. Lytchak, A. Petrunin Metric-measure boundary and geodesic flow on Alexandrov spaces, arXiv: 1705.04767.

[9] Y. Otsu, T. Shioya, The Riemannian structure of Alexandrov spaces, J. Differential Geom., 39 (1994), 629-658. 
N. Li, Department of Mathematics, The City University of New York - NYC College of Technology, 300 JAY ST., BROOKLYN, NY 11201

E-mail address: NLi@citytech. cuny.edu

A. Naber, Department Of Mathematics, Northwestern University, 2033 Sheridan Rd., Evanston, IL $60208-2370$

E-mail address: anaber@math.northwestern.edu 